\title{
Campos clássicos no plano nulo
}

\author{
Classical fields on the null-plane
}

\author{
O.A. Acevedo ${ }^{* 1}$, K.P. Gallo ${ }^{1}$, B.M. Pimentel ${ }^{1}$, G.E.R. Zambrano ${ }^{2}$ \\ ${ }^{1}$ Universidade Estadual Paulista Júlio de Mesquita Filho, Instituto de Física Teórica, São Paulo, SP, Brasil. \\ ${ }^{2}$ Universidad de Nariño, San Juan de Pasto, Colombia.
}

Recebido em 28 de maio de 2021. Revisado em 30 de junho de 2021. Aceito em 04 de julho de 2021.

\begin{abstract}
A descrição da dinâmica dos sistemas físicos requer o detalhamento da evolução das quantidades que os determinam ao passar de uma superfície tri-dimensional para outra, cada uma delas definida segundo o parâmetro de evolução a que é dado o nome de «tempo». Mas quais seriam essas superfícies e qual seria esse tempo é uma escolha não única, senão diversa, como foi mostrado por Dirac em 1949; tais diferentes escolhas possíveis inequivalentes se chamam «formas dinâmicas». No presente artigo expomos a definição precisa delas e colocamos o foco de atenção na assim chamada «forma dinâmica da frente de luz» ou do «plano nulo», em que o tempo é uma coordenada definida sobre o cone de luz. Estudamos os diversos campos clássicos livres nessa formulação: o escalar, o fermiônico, o eletromagnético e o vetorial massivo, estabelecendo a solução do problema dos valores iniciais (problema de Goursat), a distinção entre suas componentes dinâmicas e as não-dinâmicas, e seus estados de polarização. Finalizamos expondo cuidadosamente as virtudes e dificuldades da forma dinâmica do plano nulo, almejando assim fornecer um material de iniciação relevante a essa prometedora, porém pouco conhecida, área da física.
\end{abstract}

Palavras-chave: Teoria clássica de campos; dinâmica da frente de luz; dinâmica do plano nulo.

The description of the dynamics of the physical systems requires the specification of the evolution of the quantities that determine them in passing from one three-dimensional surface to the other, each one of them defined by the constant value of a parameter called «time». But such surfaces and such time can be chosen in different manners, as it was shown by Dirac in 1949; the inequivalent possible choices are called «dynamical forms». In this study we precisely define them, focusing in particular in the so-called «light-front dynamics» or «null-plane dynamics», in which the time is a coordinate defined on the light-cone. We study the classical free fields in this formulation: the scalar, fermion, electromagnetic and massive vector ones, establishing the solution to their initial-value problem (Goursat's problem), the classification of their components as dynamical and non-dynamical, and their polarization states. We finalize by highlighting the advantages and disadvantages of null-plane physics, hoping to provide a relevant initiation material to this promising, although generally unknown, area of physics.

Key-words: Classical field theory; light-front dynamics; null-plane dynamics.

\section{Introdução}

Quando se diz da descrição física de um determinado fenômeno ao que se quer indicar é ao conhecimento da evolução das grandezas observáveis que o determinam. Essas grandezas mudam segundo a dinâmica particular do fenômeno e, assim, é necessário referi-las a algum parâmetro ao que chamamos «tempo». Na nossa concepção popular, que tem lugar no espaço-tempo absoluto de Newton, o tempo é um conceito primitivo assim como o é o espaço. Disto não temos dúvida porque nessa descrição a cada tempo só pode corresponder um único valor dos observáveis, uma vez que em cada instante a partícula somente pode se encontrar em um único ponto e não em outro. Tal segurança, no entanto, é substituída pela dúvida uma vez que nos defrontamos com os conceitos

\footnotetext{
* Endereço de correspondência: oscar.acevedo@unesp.br
}

da teoria da relatividade de Poincaré-Einstein [1-4]. Esta é a teoria do espaço-tempo em que todos os sistemas inerciais de referência se equivalem, formulada de forma compatível com o fato experimentalmente provado da constância da velocidade da luz no vácuo. Ela impõe um limite às velocidades das partículas, restringe seu movimento e, em correspondência, levanta a possibilidade da foliação do espaço-tempo por superfícies diferentes daquela que usávamos na teoria newtoniana: Compensa, por assim dizer, a restrição do movimento incrementando as possibilidades de sua descrição.

O aparecimento de novas possibilidades para descrever a dinâmica o podemos entender fácilmente no contexto da mecânica do ponto, em que todas as grandezas físicas associadas a ele estão concentradas no próprio ponto e ele, então, descreve uma trajetória de evolução que é uma «linha de mundo». Em semelhante situação, a restrição que permite a completa descrição do sistema é 


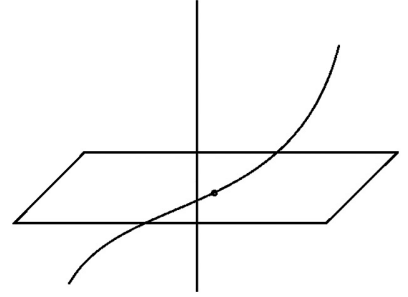

(a)

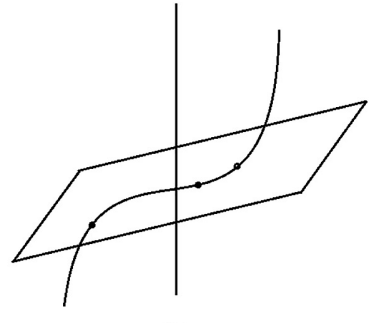

(b)
Figura 1: (a) Superfície de tempo constante. (b) Superfície inclinada.

que a mencionada linha de mundo só possa atravessar a superfície isocrônica uma única vez. Veremos a seguir quais são as superfícies que possuem esta característica.

Na dinâmica newtoniana a partícula pode ter velocidade qualquer. Assim sendo, num diagrama $t$ versus $\boldsymbol{x}$, a linha de mundo da partícula pode adotar coeficiente angular (inclinação) não-negativa qualquer 11 Portanto, é possível que uma partícula atravesse uma superfície inclinada no espaço $t-\boldsymbol{x}$ mais de uma vez, caso for acelerada suficientemente, como temos desenhado na figura 1(b), e isto acontecerá sem importar quão pequena seja a inclinação do plano. Em semelhante situação, a partícula poderia ser encontrada em mais de um ponto em cada tempo, e sua descrição se tornaria impossível. Concluindo, na dinâmica newtoniana a única forma dinâmica possível é a «dinâmica instantânea», na qual as superfícies isocrônicas são superfícies de tempo (newtoniano) constante, como mostrado na figura 11(a).

Na dinâmica relativística não enfrentamos o problema de velocidades infinitas que limitava as possíveis descrições no caso não-relativístico. Com efeito, agora a linha de mundo das partículas está restrita pela causalidade a estar sempre dentro do cone de luz, em cada ponto dela; ou seja, num diagrama $t-\boldsymbol{x}$, o coeficiente angular da linha de mundo, em cada ponto, não pode ser menor do que a unidade - ou menor do que $1 / c$, onde $c$ é a velocidade da luz no espaço vazio-. Quem primeiro se perguntou pela possibilidade de escolha das superfícies isocrônicas nessa situação foi Dirac em 1949 [6]; mais adiante detalharemos seus raciocínios. Ele conseguiu encontrar três formas dinâmicas relativísticas, a saber:

(a) Dinâmica instantânea: Aquela em que as superfícies redisocrônicas são superfícies de coordenada $x^{0}$ constante, essa coordenada sendo o tempo. Esta é a mesma forma da dinâmica newtoniana, a mais amplamente usada. Ela se mostra na figura 2(a).

(b) Dinâmica forma-ponto: Nessa forma dinâmica, que se mostra na figura 2(b), as superfícies isocrônicas são os ramos superiores dos hiperboloides $a^{2}=x^{2}$, o parâmetro $a^{2}$ sendo o tempo da teoria. Nota-

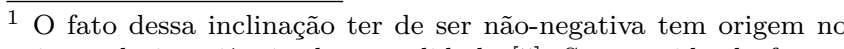
axioma de invariância da causalidade 5]: Seu sentido de futuro não pode mudar qualquer que seja sua velocidade. se que no caso limite $a^{2}=0$, os hiperboloides degeneram no cone de luz.

(c) Dinâmica da frente de luz.2 ${ }^{2}$ Aqui as superfícies isocrônicas são planos nulos de coordenada $x^{+} \sim$ $x^{0}+x^{3}$ constante, sendo ela o tempo da teoria, como se mostra na figura 2 (c).

Tal como temos apresentado, à pergunta de Dirac temos dado, como ele mesmo o fez, uma resposta intuitiva: Não temos usado um método para encontrar essas formas dinâmicas e não sabemos se elas são as únicas ou se existem outras, nem abordamos tampouco o problema de sua equivalência, de suas possibilidades e limitações. Mas já podemos perceber que a transição da multiplicidade de escolhas na dinâmica relativística à unicidade da newtoniana seria assim: $\mathrm{O}$ limite nãorelativístico das novas formas dinâmicas é a dinâmica instantânea, pois as superfícies isocrônicas, quer os hiperboloides, quer os frentes de luz, se aproximam das superficies de $x^{0}$ constante no limite $c \rightarrow+\infty$, visto que o próprio cone de luz se abre infinitamente, fazendo os hiperboloides perderem sua curvatura na dinâmica forma-ponto e os planos nulos perderem sua inclinação na dinâmica da frente de luz; por isto, o limite nãorelativístico não constitui um motivo de preferência da dinâmica instantânea sobre as outras formas dinâmicas: No mundo não-relativístico é impossível diferenciar uma da outra; a finitude de $c$ não «faz aparecer» novas formas dinâmicas, mas levanta a degenerescência entre elas.

Após o trabalho de Dirac, as formas dinâmicas por ele descobertas foram muito pouco estudadas. Mas vários anos depois, na segunda metade da década de 1960, a forma dinâmica da frente de luz reapareceu de forma totalmente independente - e com nomes diferentes como já veremos- como um método a ser usado na solução de dois problemas. Apareceu primeiramente em 1965 no trabalho de Fubini e Furlan [17] em álgebra de correntes, sob o nome de «sistema de referência de momento infinito», pois a ele tinham chegado por um processo de limite de um sistema de referência se movimentando com velocidade próxima à da luz. Este trabalho iniciou uma linha de pesquisa à qual se aderiram, por exemplo, Weinberg [18] e Bebié e Leutwyler [19. O outro problema em que essa forma dinâmica se mostrou vantajosa foi no estudo do campo do laser; isto ocorreu em 1967 durante os estudos de doutorado de Neville sob a orientação de Rohrlich [15, 20, 21. Nesses dois problemas, insistimos, a dinâmica da frente de luz foi usada como uma ferramenta para a fenomenologia, sem referência nenhuma ao artigo de Dirac. Mas a conexão entre estes trabalhos não tardou em ser reconhecida: Chang e Ma 22, em 1969, publicaram um artigo em que se estudava o modelo de interação $\varphi^{3}$, assim como a eletrodinâmica quântica

2 Algumas vezes chamada «dinâmica do cone de luz» [16], mas esse nome não é apropriado pois o cone de luz, já o dissemos, é um caso particular que corresponde à dinâmica forma-ponto, e não deve ser confundida com a dinâmica da frente de luz. 


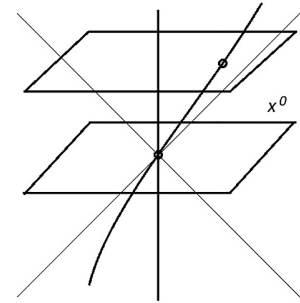

(a)

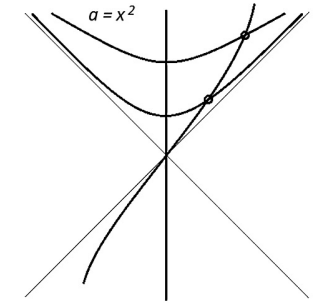

(b)

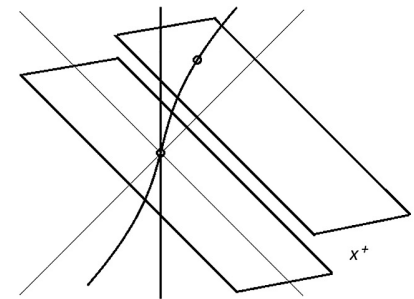

(c)

Figura 2: (a) Dinâmica instantânea. (b) Dinâmica forma-ponto. (c) Dinâmica da frente de luz.

(QED) nas novas variáveis da frente de luz; nele foi dito pela primeira vez que o sistema de referência de momento infinito correspondia-se com a forma dinâmica da frente de luz de Dirac. Por outro lado, em 1970 Kogut e Soper 23] interpretaram o passo ao sistema de referência de momento infinito como uma mudança de variáveis equivalente à formulação no plano nulo de Rohrlich e Neville. Dessarte, podemos afirmar que a dinâmica da frente de luz foi descoberta três vezes de forma independente: Primeiro em 1949 por Dirac, em 1965 por Fubini e Furlan, e em 1967 por Neville e Rohrlich, e que elas foram identificadas como uma e a mesma nos trabalhos de Chang e Ma e de Kogut e Soper.

A partir desse ponto a física no plano nulo começou a ser mais estudada: O problema dos valores iniciais para os campos clássicos foi estudado por Rohrlich e Neville [15. 24], que estabeleceram teoremas de existência e unicidade das soluções para o campo escalar de KleinGordon-Fock, para o campo de Dirac e para o campo eletromagnético sujeito à condição de gauge do plano nulo $A^{+}=0$, aduzindo importantes razões para seu uso. O plano nulo encontrou importante aplicação desde seu nascimento na teoria ondulatória (quântica), em que destacam, entre outros, o trabalho de Leutwyler, Klauder e Streit [25] sobre questões teóricas da quantização, o de Ten Eyck e Rohrlich [26, 27] e Chang, Root e Yan 28, 31] sobre a equivalência da QED no plano nulo com a QED na forma instantânea, o trabalho sobre renormalização da QED por Brodsky, Roskies e Suaya [32] e na área mais fenomenológica o uso que a ela deram Drell, Levy e Yan 33 36] no modelo a pártons.

Com o renascimento do estudo das formas dinâmicas impulsionado pela física no plano nulo advieram igualmente trabalhos de pesquisa na forma dinâmica da forma-ponto - uma revisão pode ser encontrada na Ref. 37-.

Em 1978, as três soluções encontradas por Dirac foram complementadas por importantes pesquisadores na área da física no plano nulo. Para tal foi usado o método exposto por Dirac no artigo original, que foi definir as superfícies isocrônicas em relação ao subgrupo do grupo de Poincaré que as deixa invariantes -chamado «grupo de estabilidade» da superfície-; embora Dirac tenha estabelecido suas soluções «à mão», na década de 1970 havia sido feita uma classificação dos subgrupos do grupo de Poincaré [912, o que possibilitou fazer rigoroso o método de Dirac; Leutwyler e Stern [13] encontraram mais duas formas dinâmicas, cujas superfícies isocrônicas são definidas como (d) os ramos superiores dos hiperbolóides de equação $\left(x^{0}\right)^{2}-\left(x^{1}\right)^{2}-\left(x^{2}\right)^{2}=a^{2}$, e (e) os dos hiperbolóides que seguem a lei $\left(x^{0}\right)^{2}-\left(x^{3}\right)^{2}=$ $a^{2}$; em ambos os casos, semelhantemente à forma-ponto, ao parâmetro $a^{2}$ é dada a função de tempo. Leutwyler e Stern mostraram ainda que as cinco formas dinâmicas agora conhecidas são as únicas possíveis que satisfazem a imposição da transitividade do grupo de estabilidade das superfícies isocrônicas, isto é, que todo ponto da superfície pode ser conectado com qualquer outro ponto nela por algum elemento do grupo de estabilidade. Essas novas formas dinâmicas, também, degeneram na dinâmica instantânea no limite não-relativístico.

É claro que todas essas formas dinâmicas requerem atenção e estudo, embora os autores não conheçam o trabalho realizado em alguma das duas novas formas dinâmicas adicionadas por Leutwyler e Stern. Quanto à dinâmica da frente de luz, ela possui as seguintes peculiaridades que mostraremos mais adiante: Dirac [6] mostrou que nessa forma dinâmica o número de geradores do grupo de Poincaré independentes da interação é máximo; também, os planos nulos são as superfícies características da equação diferencial de Klein-GordonFock [14, 15], equação a que todo campo deve satisfazer. Essas propriedades se oferecem à vista já na teoria clássica que é motivo do presente estudo. A esse âmbito clássico pertence também o estudo realizado por Casana, Pimentel e Zambrano [7, 8, 38] em que a estrutura de vínculos da eletrodinâmica fermiônica e a escalar, sujeitas à condição de gauge do plano nulo, é estudada na dinâmica da frente de luz usando a técnica de DiracBergmann.

As aplicações mais importantes da dinâmica da frente de luz encontram-se na física hadrônica, particularmente, para a descrição de estados compostos devido à trivialidade do seu estado de vácuo na teoria quântica [13. 16. Nesse sentido, uma técnica comumente usada é a chamada «quantização discreta no cone de luz», concebida por Maskawa e Yamawaki [39] por motivos teóri$\cos 3^{3}$ e usada na aplicação por Pauli e Brodsky [40, 41].

\footnotetext{
${ }^{3}$ Para solucionar o assim chamado «problema do modo zero».
} 
As teorias de gauge não-abelianas sob a condição de gauge do plano nulo foram estudadas por Tomboulis 42 na ausência de matéria e por Casher [43] em presença de férmions. Pimentel, Suzuki e Zambrano, na Ref. [44, estudaram também a estrutura de vínculos dessas teorias usando o método de integração funcional. Uma revisão dos avanços feitos na cromodinâmica quântica (QCD) no plano nulo em relação à física hadrônica pode ser encontrada na Ref. 45.

Contudo, há pontos na teoria do campo no plano nulo que se encontram ainda em discussão. A equivalência com a dinâmica instantânea, por exemplo, é ainda tema de debate, como é mostrado, por exemplo, na Ref. [46]. O mesmo acontece com os «termos instantâneos» que aparecem nos propagadores dos campos de Dirac e eletromagnético -vide, por exemplo, a Ref. [47-.

O presente artigo é constituído da seguinte forma. $\mathrm{Na}$ seção 2 expomos as ideias que levaram Dirac a estabelecer o problema das formas dinâmicas e a solução que a ele foi dado. Essa definição puramente algébrica é levada ao âmbito da teoria do campo clássico na seção 3 Os elementos básicos para a descrição do plano nulo são apresentados na seção 4 Na seção 5 apresentamos um breve resumo da teoria clássica do campo em forma covariante. Já as seções 6, 7, 8, e 9] estão dedicadas ao estudo dos campos escalar, fermiônico, eletromagnético e vetorial massivo no plano nulo, respectivamente. Finalmente, na seção 10 discutimos os resultados obtidos e apresentamos nossas conclusões.

\section{Definição algébrica das formas dinâmicas}

Nessa seção introduziremos as diversas formas dinâmicas seguindo as idéias apresentadas por Dirac [6], que colocou o problema da seguinte forma: Duas teorias, a relatividade de Poincaré-Einstein, que exige a invariância da física frente às transformações do grupo de Poincaré, e a mecânica quântica, cuja estrutura é hamiltoniana 4 passaram por importantes provas experimentais. Quais serão as condições gerais que permitem a descrição de um sistema físico compatível com esses dois requerimentos simultâneamente?

Comecemos com a imposição da possibilidade da formulação hamiltoniana. Essa formulação se equivale com o formalismo dos colchetes de Poisson [48, 49], segundo o qual variáveis dinâmicas quaisquer, $\xi, \eta$ e $\zeta$, devem ter colchetes de Poisson regidos pelas leis:

$$
\begin{aligned}
& \{\xi ; \eta\}=-\{\eta ; \xi\}, \\
& \{\xi ; \eta+\zeta\}=\{\xi ; \eta\}+\{\xi ; \zeta\},
\end{aligned}
$$

\footnotetext{
4 Naquela época, é mister dizer, a formulação hamiltoniana era imprescindível para a transição da teoria clássica à quântica - assim o apontou Dirac explícitamente-; na atualidade tal restrição não rege mais e é perfeitamente possível formular a teoria quântica desde a abordagem lagrangiana ou ainda diretamente a partir das equações do movimento dos campos.
}

$$
\begin{aligned}
& \{\xi ; \eta \zeta\}=\{\xi ; \eta\} \zeta+\eta\{\xi ; \zeta\}, \\
& \{\{\xi ; \eta\} ; \zeta\}+\{\{\eta ; \zeta\} ; \xi\}+\{\{\zeta ; \xi\} ; \eta\}=0 .
\end{aligned}
$$

Mas as variáveis dinâmicas estão definidas em relação ao sistema das coordenadas - exemplo disto são as próprias coordenadas e os momentos-, de forma que aquelas mudam quando essas são submetidas a uma transformação. Sob a transformação infinitesimal das coordenadas, cada variável dinâmica $\xi$ transforma-se segundo:

$$
\xi^{\prime}=\xi+\{\xi ; F\}
$$

com $F$ alguma variável dinâmica infinitesimal, dependente apenas do sistema envolvido e da transformação de coordenadas, e ao que se dá o nome de «gerador da transformação». Essa forma da variação garante que as relações entre os colchetes de Poisson das diversas variáveis dinâmicas permaneçam inalteradas, o que permite cumprir a exigência da teoria da relatividade: «Sob as transformações do grupo de Poincaré, as variações das variáveis dinâmicas devem ser tais que os colchetes de Poisson entre elas permaneçam os mesmos». Assim, se faz necessário examinar os geradores das transformações de Poincaré. Uma transformação infinitesimal de Poincaré muda as coordenadas segundo:

$$
x^{\prime \mu}=x^{\mu}+a^{\mu}+b^{\mu}{ }_{\nu} x^{\nu},
$$

com $a^{\mu}$ os parâmetros infinitesimais da translação e $b_{\mu \nu}=-b_{\nu \mu}$ os parâmetros infinitesimais associados à transformação de Lorentz. Mantendo a discussão em nível infinitesimal, o gerador $F$ deverá também ser linear nestes parâmetros:

$$
F=-P_{\mu} a^{\mu}+\frac{1}{2} M_{\mu \nu} b^{\mu \nu} ; \quad M_{\mu \nu}=-M_{\nu \mu} .
$$

A dependência com a transformação está inclusa nos parâmetros $a$ e $b$; logo as variáveis dinâmicas $P$ e $M$ caracterizam o sistema em estudo. Elas se chamam «quantidades fundamentais», e estão sujeitas à álgebra do grupo de Poincaré, como o demonstramos no apêndice $\mathrm{A}$.

$$
\begin{aligned}
& \left\{P_{\mu} ; P_{\nu}\right\}=0, \\
& \left\{M_{\mu \nu} ; P_{\rho}\right\}=-g_{\mu \rho} P_{\nu}+g_{\nu \rho} P_{\mu}, \\
& \left\{M_{\mu \nu} ; M_{\rho \sigma}\right\}=-g_{\mu \rho} M_{\nu \sigma}+g_{\nu \rho} M_{\mu \sigma}-g_{\mu \sigma} M_{\rho \nu} \\
& \quad+g_{\nu \sigma} M_{\rho \mu} .
\end{aligned}
$$

Neste ponto, Dirac introduz a noção do que sejam as formas dinâmicas: Elas são as soluções para as relações algébricas que acabamos de escrever. Para entender o que isto quer dizer, lembre o leitor que nem todas as transformações do grupo de simetrias do espaço-tempo levam à evolução dinâmica das superfícies isocrônicas; na teoria newtoniana, por exemplo, em que o grupo de simetrias é o grupo de Galilei, uma translação espacial ou uma rotação não muda a superfície de tempo constante, 
apenas a transforma em si mesma, são «transformações cinemáticas» e a superfície isocrônica é estável em relação a elas. O mesmo ocorre na teoria relativística: Alguns dos geradores darão lugar a transformações cinemáticas e constituirão o «grupo de estabilidade» das superfícies isocrônicas, mapeando estas em si mesmas; os outros geradores levam a transformações que mudam a superfície, a fazem evoluir, e assim devem ser identificados com as transformações dinâmicas; a estes geradores Dirac dá o nome de «hamiltonianos».

A partir daqui, já é evidente que o grupo de estabilidade e os hamiltonianos dependerão de qual seja a superfície isocrônica escolhida, e daí o interesse nas diversas formas dinâmicas: Pode acontecer -como de fato acontece, segundo comentamos na seção 1- que para alguns problemas a descrição da dinâmica seja simplificada em uma determinada forma dinâmica, pois os hamiltonianos podem ser mais simples ou mais complicados segundo a escolha da superfície isocrônica. Se tais grupos de estabilidade não são isomórficos, então as formas dinâmicas que lhes correspondem não poderão ser deformadas uma na outra por uma simples transformação de Poincaré; em tais situações, que são precisamente as que definem formas dinâmicas «diferentes», a equivalência física da descrição não se deriva do axioma da relatividade: Esse é o problema da equivalência.

Para relacionar as superfícies isocrônicas com seu grupo de estabilidade, as descreveremos por meio de uma função $\Sigma=\Sigma(x)$ cujo valor é constante nas superfícies, ou seja, ele é a expressão do «tempo» da forma dinâmica. Os geradores do grupo de estabilidade serão aqueles que satisfaçam:

$$
\{\Sigma(x) ; F\}=0
$$

pois assim sendo, segundo a equação (5), a transformação gerada por $F$ deixa invariante o valor do tempo $\Sigma$. Os geradores que não satisfazem à equação (11), por outra parte, serão os geradores da dinâmica. O colchete da equação (11) pode ser calculado uma vez que seja conhecida a função $\Sigma(x)$ pelo uso das equações 292 .

Forma instantânea. Nessa forma dinâmica as superfícies isocrônicas são aquelas em que a coordenada $x^{0}$ se mantém constante:

$$
\Sigma(x)=x^{0} .
$$

Usando as equações 292 vemos que:

$$
\left\{x^{0} ; P_{\rho}\right\}=-\delta_{\rho}^{0}, \quad\left\{x^{0} ; M_{\rho \sigma}\right\}=\delta_{\rho}^{0} x_{\sigma}-\delta_{\sigma}^{0} x_{\rho} .
$$

O grupo de estabilidade dessas superfícies espaciais contém, como na física newtoniana, as translações e rotações espaciais, correspondentes aos geradores $P_{1}, \quad P_{2}, \quad P_{3}, M_{12}, \quad M_{23}$ e $M_{31}$, que são, então, os geradores cinemáticos da forma instantânea. Que eles constituem uma sub-álgebra do grupo de Poincaré -e então dão origem a um de seus subgrupos- pode ser diretamente verificado com o uso das equações (8)-(10): Todos os colchetes de Poisson desses geradores têm por resultado uma expressão que contém a eles mesmos só. Os outros geradores, a saber, $P_{0}, M_{10}, M_{20}$ e $M_{30}$, são os geradores dinâmicos ou hamiltonianos. Assim, o grupo de estabilidade da forma instantânea tem dimensão sêis, e possui quatro hamiltonianos.

Forma-ponto. Na forma-ponto o tempo é dado pela função:

$$
\Sigma(x)=x^{\mu} x_{\mu}
$$

Calculando a ação dos geradores das transformações de Poincaré sobre ela:

$$
\left\{x^{\mu} x_{\mu} ; P_{\rho}\right\}=-2 x_{\rho}, \quad\left\{x^{\mu} x_{\mu} ; M_{\rho \sigma}\right\}=0 .
$$

Portanto, o grupo de estabilidade é constituído pelas transformações geradas por $M_{\mu \nu}$ e tem dimensão seis, ao passo que os geradores $P_{\mu}$ serão os quatro hamiltonianos da forma dinâmica. Embora sejam em número igual que na forma instantânea, a forma-ponto possui a vantagem de apresentar hamiltonianos que compõem um tetravetor, podendo ser tratados como uma única entidade covariante. A nomeação de «forma-ponto» dada a essa dinâmica é devida à seguinte propriedade: Que seu grupo de estabilidade é o grupo de rotações tetra-dimensionais ao redor de um ponto fixo.

Forma da frente de luz. Nesta forma dinâmica a superfície isocrônica é a superfície de valor constante do tempo

$$
\Sigma(x)=x^{+}=\frac{1}{\sqrt{2}}\left(x^{0}+x^{3}\right) .
$$

Para ela obtemos que:

$$
\left\{x^{+} ; P_{\rho}\right\}=-\delta_{\rho}^{+}, \quad\left\{x^{+} ; M_{\rho \sigma}\right\}=\delta_{\rho}^{+} x_{\sigma}-\delta_{\sigma}^{+} x_{\rho} .
$$

Ocorre aqui algo curioso: Somente se a superfície for a $x^{+}=0$, o colchete de Poisson $\left\{x^{+} ; M_{+-}\right\}$se anula, como se observa da equação 17$) \cdot M_{+-}$, portanto, forma parte do grupo de estabilidade da superfície $x^{+}=0$, mas não das superfícies correspondentes a outros tempos. Restringindo nossa atenção a essa superfície particular, que sempre pode ser usada como a superfície inicial no problema de valores iniciais, teremos que o grupo de estabilidade tem dimensão sete e é formada pelos geradores $P_{1}, P_{2}, P_{-}, M_{12}, M_{1-}, M_{2-}$ e $M_{+-}$. O número de hamiltonianos é três, e são $P_{+}, M_{1+}$ e $M_{2+}$.

Forma de Leutwyler-Stern 1. Na primeira forma de Leutwyler e Stern a superfície isocrônica é definida pelo tempo:

$$
\Sigma(x)=\left(x^{0}\right)^{2}-\left(x^{1}\right)^{2}-\left(x^{2}\right)^{2}=x^{\mu} x_{\mu}+\left(x^{3}\right)^{2} .
$$


A ação dos geradores do grupo de Poincaré sobre ela é:

$$
\begin{aligned}
& \left\{x^{\mu} x_{\mu}+\left(x^{3}\right)^{2} ; P_{\rho}\right\}=-2 x_{\rho}-2 x^{3} \delta_{\rho}^{3}, \\
& \left\{x^{\mu} x_{\mu}+\left(x^{3}\right)^{2} ; M_{\rho \sigma}\right\}=2 x^{3}\left(\delta_{\rho}^{3} x_{\sigma}-\delta_{\sigma}^{3} x_{\rho}\right) .
\end{aligned}
$$

Vemos assim que o grupo de estabilidade tem apenas dimensão quatro, e a ele correspondem os geradores $P_{3}$, $M_{01}, M_{02}$ e $M_{12}$. Os hamiltonianos dessa forma dinâmica são seis: $P_{0}, P_{1}, P_{2}, M_{03}, M_{13}$ e $M_{23}$.

Forma de Leutwyler-Stern 2. A segunda forma introduzida por Leutwyler e Stern possui o tempo definido segundo a função:

$$
\Sigma(x)=\left(x^{0}\right)^{2}-\left(x^{3}\right)^{2} .
$$

Com o uso das equações 292 obtemos que:

$$
\begin{gathered}
\left\{\left(x^{0}\right)^{2}-\left(x^{3}\right)^{2} ; P_{\rho}\right\}=-2 x^{0} \delta_{\rho}^{0}+2 x^{3} \delta_{\rho}^{3}, \\
\left\{\left(x^{0}\right)^{2}-\left(x^{3}\right)^{2} ; M_{\rho \sigma}\right\}=2 x^{0}\left(\delta_{\rho}^{0} x_{\sigma}-\delta_{\sigma}^{0} x_{\rho}\right) \\
-2 x^{3}\left(\delta_{\rho}^{3} x_{\sigma}-\delta_{\sigma}^{3} x_{\rho}\right) .
\end{gathered}
$$

Com esses resultados podemos estabelecer que o grupo de estabilidade das superfícies de $\Sigma$ constante é aquele correspondente aos geradores $P_{1}, P_{2}, M_{12}$ e $M_{03}$, e portanto tem dimensão quatro. Os seis hamiltonianos dessa forma dinâmica serão os geradores $P_{0}, P_{3}, M_{01}$, $M_{02}, M_{13}$ e $M_{23}$.

E isso esgota as possibilidades, uma vez que não há outro subgrupo do grupo de Poincaré com a propriedade da transitividade que possa servir como grupo de estabilidade de alguma outra superfície [9]13. Os resultados que assim obtivemos da classificação algébrica das cinco possíveis formas dinâmicas na teoria relativística se resumem na tabela 1. Como podemos ver, a dinâmica da frente de luz ocupa um lugar destacado por ser a forma dinâmica com o maior grupo de estabilidade e, consequentemente, aquele com o menor número de hamiltonianos.

\subsection{Mecânica do ponto material}

Uma primeira solução, a mais simples, às equações (8) - 10 é obtida na mecânica do ponto material:

$$
P_{\mu}=p_{\mu}, \quad M_{\mu \nu}=q_{\mu} p_{\nu}-q_{\nu} p_{\mu},
$$

Tabela 1: Resumo das cinco formas dinâmicas da teoria relativística. $\mathrm{D}\left(G_{\Sigma}\right)$ : Dimensão do grupo de estabilidade $G_{\Sigma}$; N. de H's: Número de hamiltonianos.

\begin{tabular}{lccc}
\hline F. dinâmica & $\Sigma(x)$ & $\mathrm{D}\left(G_{\Sigma}\right)$ & N. de H's \\
\hline Instantânea & $x^{0}$ & 6 & 4 \\
Forma-ponto & $x^{\mu} x_{\mu}$ & 6 & 4 \\
Frente de luz & $x^{+}$ & 7 & 3 \\
L-S 1 & $x^{\mu} x_{\mu}+\left(x^{3}\right)^{2}$ & 4 & 6 \\
L-S 2 & $\left(x^{0}\right)^{2}-\left(x^{3}\right)^{2}$ & 4 & 6 \\
\hline
\end{tabular}

com as coordenadas $q_{\mu}$ e momentos $p_{\mu}$ da partícula sujeitos a:

$$
\left\{q_{\mu} ; q_{\nu}\right\}=0,\left\{p_{\mu} ; p_{\nu}\right\}=0,\left\{p_{\mu} ; q_{\nu}\right\}=g_{\mu \nu} .
$$

Desejamos em cada forma dinâmica escrever expressões para esses geradores sobre a superfície inicial (isocrônica). Para isto será necessário incorporar as equações que determinam tal superfície como equações subsidiárias, do tipo:

$$
A \approx 0
$$

Mas as condições subsidiárias devem permanecer tais frente a qualquer mudança do sistema de coordenadas, o que implica que devam também ser condições subsidiárias:

$$
\left\{A ; P_{\rho}\right\} \approx 0, \quad\left\{A ; M_{\rho \sigma}\right\} \approx 0 .
$$

Para eliminar as variáveis cujo significado é perdido ao fixar a superfície inicial, usaremos a condição de camada de massa, $p^{2}-m^{2}=0$, mas a introduziremos nos geradores do grupo de Poincaré usando coeficientes indeterminados (multiplicadores de Lagrange); as equações (22) serão substituídas por:

$$
\begin{aligned}
& P_{\mu}=p_{\mu}+\lambda_{\mu}\left(p^{2}-m^{2}\right), \\
& M_{\mu \nu}=q_{\mu} p_{\nu}-q_{\nu} p_{\mu}+\lambda_{\mu \nu}\left(p^{2}-m^{2}\right),
\end{aligned}
$$

com $\lambda_{\mu \nu}=-\lambda_{\nu \mu}$. Tais coeficientes serão determinados pela imposição da equação 25.

Forma instantânea. Temos a condição subsidiária:

$$
q^{0} \approx 0
$$

A anulação do seu colchete de Poisson com o gerador $P_{\mu}$ somente acontece se:

$$
\left\{q^{0} ; P_{\sigma}\right\}=-\delta_{\sigma}^{0}-2 \lambda_{\sigma} p_{0} \approx 0,
$$

enquanto a equação correspondente às transformações de Lorentz é:

$$
\left\{q^{0} ; M_{\rho \sigma}\right\}=-q_{\rho} \delta_{\sigma}^{0}+q_{\sigma} \delta_{\rho}^{0}-\lambda_{\rho \sigma} 2 p_{0} \approx 0 .
$$

Das equações 29$)$ e 30 decorrem os valores:

$$
\lambda_{0}=-\frac{1}{2 p_{0}}, \lambda_{r}=0, \lambda_{r 0}=-\frac{q_{r}}{2 p_{0}}, \lambda_{r s}=0,
$$

$r, s=1,2,3$. Os geradores na superfície inicial são portanto:

$$
\begin{aligned}
& P_{0}=\sqrt{\boldsymbol{p}^{2}+m^{2}}, \quad P_{r}=p_{r}, \\
& M_{r 0}=q_{r} \sqrt{\boldsymbol{p}^{2}+m^{2}}, \quad M_{r s}=q_{r} p_{s}-q_{s} p_{r} .
\end{aligned}
$$

Temos usado aqui a notação $\boldsymbol{p}^{2}=p_{1}^{2}+p_{2}^{2}+p_{3}^{2}$. 
Forma-ponto. A condição subsidiária nessa forma dinâmica é:

$$
q^{\mu} q_{\mu} \approx \kappa^{2}=\text { constante. }
$$

Essa constante em geral será não nula; porém, ela pode também ser nula, caso em que o hiperboloide degenera no cone de luz. As condições da equação 25 são assim:

$$
\begin{aligned}
& \left\{q^{\mu} q_{\mu} ; P_{\rho}\right\}=-2 q_{\rho}-4 \lambda_{\rho} q \cdot p \approx 0, \\
& \left\{q^{\mu} q_{\mu} ; M_{\rho \sigma}\right\}=-4 \lambda_{\mu \nu} q \cdot p \approx 0 .
\end{aligned}
$$

Dessas equações, obtemos o valor dos coeficientes indeterminados:

$$
\lambda_{\rho}=-\frac{q_{\rho}}{2 q \cdot p}, \quad \lambda_{\mu \nu}=0,
$$

e assim também o dos geradores do grupo de Poincaré:

$$
\begin{aligned}
& P_{\mu}=p_{\mu}-\frac{q_{\mu}\left(p^{2}-m^{2}\right)}{2 q \cdot p}, \\
& M_{\mu \nu}=q_{\mu} p_{\nu}-q_{\nu} p_{\mu} .
\end{aligned}
$$

Forma da frente de luz. A condição subsidiária consiste, dessa vez, na especificação do plano nulo inicial:

$$
q^{+} \approx 0
$$

Agora temos as restrições:

$$
\begin{aligned}
& \left\{q^{+} ; P_{\rho}\right\}=-\delta_{\rho}^{+}-2 \lambda_{\rho} p_{-} \approx 0, \\
& \left\{q^{+} ; M_{\rho \sigma}\right\}=\delta_{\rho}^{+} q_{\sigma}-\delta_{\sigma}^{+} q_{\rho}-2 \lambda_{\rho \sigma} p_{-} \approx 0 .
\end{aligned}
$$

Delas obtemos os multiplicadores -usamos $i, j=1,2-$ :

$$
\begin{aligned}
& \lambda_{+}=-\frac{1}{2 p_{-}}, \lambda_{i}=0=\lambda_{-}, \lambda_{+-}=0, \\
& \lambda_{i+}=-\frac{q_{i}}{2 p_{-}}, \lambda_{i j}=0, \lambda_{i-}=0 .
\end{aligned}
$$

E, daqui, os geradores do grupo de Poincaré no plano nulo $x^{+}=0$ são:

$$
\begin{aligned}
& P_{+}=\frac{p_{\perp}^{2}+m^{2}}{2 p_{-}}, P_{-}=p_{-}, P_{i}=p_{i}, \\
& M_{i+}=q_{i} \frac{p_{\perp}^{2}+m^{2}}{2 p_{-}}-q_{+} p_{i}, M_{+-}=q_{+} p_{-}, \\
& M_{12}=q_{1} p_{2}-q_{2} p_{1}, M_{i-}=q_{i} p_{-} .
\end{aligned}
$$

Nestas expressões: $p_{\perp}^{2}=p_{1}^{2}+p_{2}^{2}$.

Forma de Leutwyler-Stern 1. Na primeira forma de Leutwyler-Stern a condição subsidiária imposta às coordenadas da partícula é:

$$
q^{\mu} q_{\mu}+\left(q^{3}\right)^{2} \approx \kappa^{2}=\text { constante }
$$

Encontramos as seguintes condições adicionais:

$$
\begin{aligned}
& \left\{q^{\mu} q_{\mu}+\left(q^{3}\right)^{2} ; P_{\rho}\right\} \\
& =-2 q_{\rho}-2 q^{3} \delta_{\rho}^{3}-4 \lambda_{\rho}\left(q^{\mu} p_{\mu}+q^{3} p^{3}\right) \approx 0 \\
& \left\{q^{\mu} q_{\mu}+\left(q^{3}\right)^{2} ; M_{\rho \sigma}\right\} \\
& 2 q^{3}\left(\delta_{\rho}^{3} q_{\sigma}-\delta_{\sigma}^{3} q_{\rho}\right)-4 \lambda_{\mu \nu}\left(q^{\mu} p_{\mu}+q^{3} p^{3}\right) \approx 0,
\end{aligned}
$$

das quais podemos isolar os coeficientes indeterminados:

$$
\begin{aligned}
& \lambda_{0,1,2}=-\frac{q_{0,1,2}}{2\left(q^{\mu} p_{\mu}+q^{3} p^{3}\right)}, \lambda_{3}=0, \\
& \lambda_{01}=\lambda_{02}=\lambda_{12}=0, \lambda_{(0,1,2) 3}=-\frac{q_{0,1,2} q^{3}}{2\left(q^{\mu} p_{\mu}+q^{3} p^{3}\right)} .
\end{aligned}
$$

As quantidades fundamentais nesta forma dinâmica são portanto:

$$
\begin{aligned}
& P_{0,1,2}=p_{0,1,2}-\frac{q_{0,1,2}\left(p^{2}-m^{2}\right)}{2\left(q^{\mu} p_{\mu}+q^{3} p^{3}\right)}, P_{3}=p_{3}, \\
& M_{01}=q_{0} p_{1}-q_{1} p_{0}, M_{02}=q_{0} p_{2}-q_{2} p_{0}, \\
& M_{12}=q_{1} p_{2}-q_{2} p_{1}, \\
& M_{(0,1,2) 3}=q_{0,1,2} p_{3}-q_{3} p_{0,1,2}-\frac{q_{0,1,2} q^{3}\left(p^{2}-m^{2}\right)}{2\left(q^{\mu} p_{\mu}+q^{3} p^{3}\right)} .
\end{aligned}
$$

Forma de Leutwyler-Stern 2. Nesta última forma dinâmica a condição subsidiária adota a forma:

$$
\left(q^{0}\right)^{2}-\left(q^{3}\right)^{2} \approx \kappa^{2}=\text { constante. }
$$

As condições que tal restrição implica são as que seguem:

$$
\begin{aligned}
& \left\{\left(q^{0}\right)^{2}-\left(q^{3}\right)^{2} ; P_{\rho}\right\} \\
& =-2 q^{0} \delta_{\rho}^{0}+2 q^{3} \delta_{\rho}^{3}-4 \lambda_{\rho}\left(q^{0} p^{0}-q^{3} p^{3}\right) \approx 0, \\
& \left\{\left(q^{0}\right)^{2}-\left(q^{3}\right)^{2} ; M_{\rho \sigma}\right\}=2 q^{0}\left(\delta_{\rho}^{0} q_{\sigma}-\delta_{\sigma}^{0} q_{\rho}\right) \\
& \quad-2 q^{3}\left(\delta_{\rho}^{3} q_{\sigma}-\delta_{\sigma}^{3} q_{\rho}\right)-4 \lambda_{\rho \sigma}\left(q^{0} p^{0}-q^{3} p^{3}\right) \approx 0 .
\end{aligned}
$$

Desse sistema, obtemos os valores dos multiplicadores de Lagrange:

$$
\begin{aligned}
& \lambda_{0}=-\frac{q^{0}}{2\left(q^{0} p^{0}-q^{3} p^{3}\right)}, \lambda_{1,2}=0, \\
& \lambda_{3}=\frac{q^{3}}{2\left(q^{0} p^{0}-q^{3} p^{3}\right)}, \lambda_{0(1,2)}=\frac{q^{0} q_{1,2}}{2\left(q^{0} p^{0}-q^{3} p^{3}\right)}, \\
& \lambda_{03}=0, \lambda_{12}=0, \lambda_{(1,2) 3}=\frac{q^{3} q_{1,2}}{2\left(q^{0} p^{0}-q^{3} p^{3}\right)} .
\end{aligned}
$$

Os geradores ficam da seguinte forma:

$$
\begin{aligned}
& P_{0}=p_{0}-\frac{q^{0}\left(p^{2}-m^{2}\right)}{2\left(q^{0} p^{0}-q^{3} p^{3}\right)}, P_{1,2}=p_{1,2}, \\
& P_{3}=p_{3}+\frac{q^{3}\left(p^{2}-m^{2}\right)}{2\left(q^{0} p^{0}-q^{3} p^{3}\right)},
\end{aligned}
$$




$$
\begin{aligned}
& M_{0(1,2)}=q_{0} p_{1,2}-q_{1,2} p_{0}+\frac{q^{0} q_{1,2}\left(p^{2}-m^{2}\right)}{2\left(q^{0} p^{0}-q^{3} p^{3}\right)}, \\
& M_{03}=q_{0} p_{3}-q_{3} p_{0}, M_{12}=q_{1} p_{2}-q_{2} p_{1}, \\
& M_{(1,2) 3}=q_{1,2} p_{3}-q_{3} p_{1,2}+\frac{q^{3} q_{1,2}\left(p^{2}-m^{2}\right)}{2\left(q^{0} p^{0}-q^{3} p^{3}\right)} .
\end{aligned}
$$

Como podemos observar, em todas as formas dinâmicas os geradores do grupo de estabilidade da superfície inicial não são modificados pela restrição a ela, mas somente os hamiltonianos o são, em correspondência com a sua ação. Poderemos nos perguntar agora, nesta situação que é ainda a mais simples possível, que é a do ponto material livre, qual é a forma dinâmica que se apresenta mais vantajosa? Olhando para as expressões que acabamos de escrever para os hamiltonianos, é claro que as formas de Leutwyler-Stern não o serão, não já somente pela complexidade de seus hamiltonianos, mas também pelo fato de serem seis deles. A forma instantânea, muito usada, apresenta a dificuldade de uma raíz quadrada; em relação a este ponto, lembre o leitor a impossibilidade de escrever a equação de Klein-Gordon-Fock na forma hamiltoniana na classe dos operadores diferenciais; isto último somente é possível ao ampliar dita classe e considerar os operadores pseudo-diferenciais -o leitor interessado pode consultar a Ref. [50]-. Semelhante dificuldade é evitada na formaponto e na forma da frente de luz, que se mostram assim muito convenientes.

Já indicamos anteriormente uma vantagem importante da forma-ponto, que é o fato de seus hamiltonianos constituirem um tetra-vetor: A covariância é fácilmente implementada nessa forma dinâmica. No entanto, na expressão de seus hamiltonianos aparece sempre o produto $q \cdot p$ no denominador, o que faz com que eles sejam complicados. Comparativamente, na forma do plano nulo, além de ser aquela em que o número de hamiltonianos é menor, eles têm também uma forma bastante simples; e em adição seu hamiltoniano $P_{+}$, associado à energia, depende única e exclusivamente dos momentos, a posição não aparece nele, diferentemente da forma-ponto. Convencidos por estes motivos do interesse da forma dinâmica da frente de luz, passaremos a considerar já não a mecânica do ponto, mas os campos clássicos construidos nela.

\section{Forma dinâmica do plano nulo e campos clássicos}

A discussão que acabamos de fazer a respeito da possibilidade de definir novas formas dinâmicas tem sido baseada na álgebra do grupo de Poincaré e exemplificada na mecânica do ponto material. E para tal, foi suficiente exigir a descrição unívoca de sua linha de mundo. No entanto, na teoria de campos isto não é suficiente, e devemos nos ocupar também da unicidade da descrição da evolução do campo, isto é, do problema de valores iniciais para as diferentes equações do campo. Com efeito:
A estrutura causal da teoria da relatividade implica que o valor adotado pelo campo em cada ponto do espaçotempo depende dos valores que ele tem adotado no cone de luz passado correspondente a tal ponto; donde a inicialização dos valores do campo em uma superfície isocrônica requer conhecer os valores do campo na interseção do referido cone de luz passado e a superfície inicial. Isto é mostrado para as três formas dinâmicas de Dirac na figura 3. Mas logo percebemos uma dificuldade na forma dinâmica do plano nulo: Enquanto que a interseção é uma região finita na forma instantânea e na forma-ponto, ela é uma região infinita na forma do plano nulo. Sendo assim, o problema dos valores iniciais no plano nulo não poderá ser abordado como uma simples transformação do problema de Cauchy, mas precisa ser estudado independentemente. Eis o objetivo da presente seção.

$\mathrm{Na}$ teoria relativística, a invariância sob translações implica que a equação que governa todas as componentes $u(x)$ de todos os campos é a equação de Klein-GordonFock:

$$
\left(\square+m^{2}\right) u(x)=0 .
$$

Qualquer relação adicional entre as diferentes componentes de um mesmo campo constitui uma relação de vínculo, mas ela não impede o cumprimento da equação (56) -um caso especial, que pareceria contradizer o dito e que contudo não o faz, é o do campo eletromagnético, que estudaremos na seção 8- Lembremos ao leitor que a obtenção dessa equação obedece ao fato de que os campos se transformam por representações do grupo de Poincaré, um de cujos operadores de Casimir é o quadrado do operador de momento: $P^{2}$, então proporcional à identidade (com fator de proporcionalidade que temos denotado por $\mathrm{m}^{2}$ ), como o assegura o lema de Schur.

Determinar quais dados são os que se precisa conhecer para poder antecipar o valor do campo em um ponto futuro é o problema dos valores iniciais, que para equações diferenciais de segunda ordem pode ser precisamente formulado da seguinte forma 51:

\section{Problema dos valores iniciais: Seja}

$$
\phi\left(u ; x_{i} ; \frac{\partial u}{\partial x_{i}} ; \frac{\partial^{2} u}{\partial x_{i}^{2}} ; \frac{\partial^{2} u}{\partial x_{i} \partial x_{k}}\right)=0 \quad(i=1, \cdots, m)
$$

uma equação diferencial parcial de segunda ordem, linear em $u$ e suas derivadas com coeficientes que são funções quaisquer das variáveis $x_{i}$. O problema dos valores iniciais com respeito à «superfície inicial» $x_{m}=0$ consiste em encontrar uma solução sujeita aos «dados iniciais»:

$$
\begin{aligned}
& u\left(x_{1} ; \cdots ; x_{m-1} ; 0\right)=u_{0}\left(x_{1} ; \cdots ; x_{m-1}\right), \\
& \frac{\partial u}{\partial x_{m}}\left(x_{1} ; \cdots ; x_{m-1} ; 0\right)=u_{1}\left(x_{1} ; \cdots ; x_{m-1}\right) .
\end{aligned}
$$

É claro, toda equação diferencial, quer ordinária, quer parcial, admite um número infinito de soluções, 


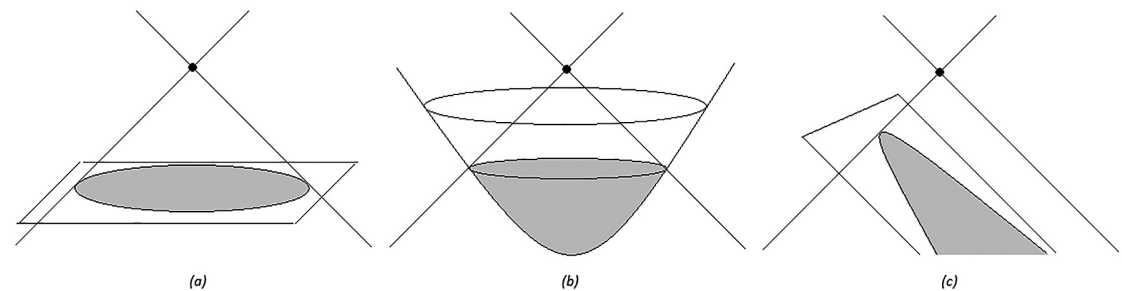

Figura 3: Problema dos valores iniciais: (a) Na dinâmica instantânea, (b) na dinâmica forma-ponto, (c) na dinâmica da frente de luz.

que são escritas como uma «integral geral» contendo um determinado número de parâmetros ou funções desconhecidas. O problema dos valores iniciais inclui em seu enunciado um conjunto de condições adicionais os dados iniciais- que visam determinar os elementos arbitrários da integral geral, mas não é, em princípio, claro que seja possível com elas determinar uma e só uma solução, pois pode acontecer que os dados iniciais sejam incompatíveis com a integral geral ou que não sejam suficientes para fixar todos os elementos arbitrários. Assim, diremos que o problema dos valores iniciais está bem definido se ele (i) tem solução, e (ii) dita solução é única.

\subsection{Problema de Cauchy-Kovalevskaya}

O caso mais famoso do problema dos valores iniciais é o "problema de Cauchy», que se caracteriza porque na equação diferencial a segunda derivada de $u$ em relação à variável $x_{m}$ que define a superfície inicial $x_{m}=0$ possui um coeficiente não nulo. As condições sob as quais o problema de Cauchy está bem definido são estabelecidas no seguinte teorema, que apresentaremos para o caso de duas variáveis independentes $x$ e $y-0$ que é suficiente para ilustrar o procedimento de solução e suas dificuldades-:

Teorema de Cauchy-Kovalevskaya: Seja o problema de valores iniciais para a equação diferencial parcial de segunda ordem e duas variáveis

$$
\phi\left(u ; x ; y ; \frac{\partial u}{\partial x} ; \frac{\partial u}{\partial y} ; \frac{\partial^{2} u}{\partial x^{2}} ; \frac{\partial^{2} u}{\partial y^{2}} ; \frac{\partial^{2} u}{\partial x \partial y}\right)=0 .
$$

Se acontece que

$$
\frac{\partial \phi}{\partial\left(\partial^{2} u / \partial x^{2}\right)} \neq 0
$$

de tal forma que podemos escrever a equação (59) na forma:

$$
\frac{\partial^{2} u}{\partial x^{2}}=f\left(u ; x ; y ; \frac{\partial u}{\partial x} ; \frac{\partial u}{\partial y} ; \frac{\partial^{2} u}{\partial y^{2}} ; \frac{\partial^{2} u}{\partial x \partial y}\right),
$$

com $f$ uma função holomórfica -isto é, analítica e regular- na vizinhança de $x=0$, então o problema de Cauchy está bem definido para qualquer valor funcional dos dados de Cauchy:

$$
u(0 ; y)=u_{0}(y) \quad \text { e } \quad \frac{\partial u}{\partial x}(0 ; y)=u_{1}(y)
$$

O leitor interessado nos detalhes técnicos desse teorema pode consultar as Refs. [51, 52. O que aqui exporemos é a construção da solução, que tem como consequência direta a sua unicidade. Escrevamos a função incógnita $u(x ; y)$ como série de potências na variável $x$, ao redor do ponto $x=0$ em que são definidos os dados de Cauchy:

$$
\begin{aligned}
& u=u_{0}+u_{1} x+\cdots+\frac{u_{h}}{h !} x^{h}+\cdots ; \\
& u_{h}=\left.u_{h}(y) \equiv \frac{\partial^{h} u}{\partial x^{h}}\right|_{x=0} .
\end{aligned}
$$

Nesta série, os dados de Cauchy já estão introduzidos como os elementos $u_{0}$ e $u_{1}$, portanto, devemos nos preocupar em encontrar $u_{h}$ para $h \geq 2$ em função deles. Notando que, em geral:

$$
\left.\frac{\partial^{h+k} u}{\partial x^{h} \partial y^{k}}\right|_{x=0}=\frac{\partial^{k} u_{h}}{\partial y^{k}},
$$

podemos obter a solução desejada com o seguinte conjunto de passos: Tomando $x=0$ na equação 61 obtemos:

$$
u_{2}=f\left(u_{0} ; 0 ; y ; u_{1} ; \frac{\partial u_{0}}{\partial y} ; \frac{\partial^{2} u_{0}}{\partial y^{2}} ; \frac{\partial u_{1}}{\partial y}\right),
$$

o que significa que $u_{2}$ está determinado pelo conhecimento de $u_{0}$ e $u_{1}$. O fato de aparecer também suas derivadas na equação 65 não é motivo de preocupação, pois elas estão tomadas em relação à variável $y$ com $x=0$, e, portanto, podem se obter dos dados de Cauchy. Derivemos agora a equação (61) em relação a $x$, então coloquemos $x=0$; obteremos:

$$
\begin{aligned}
u_{3}= & \frac{\partial f}{\partial u} u_{1}+\frac{\partial f}{\partial x}+\frac{\partial f}{\partial(\partial u / \partial x)} u_{2}+\frac{\partial f}{\partial(\partial u / \partial y)} \frac{\partial u_{1}}{\partial y} \\
& +\cdots+\frac{\partial f}{\partial\left(\partial^{2} u / \partial x \partial y\right)} \frac{\partial u_{2}}{\partial y},
\end{aligned}
$$

todas essas derivadas tomadas nos argumentos de $f$ na equação (65). Assim, a equação (66) diz que a função $u_{3}$ está determinada pelos dados de Cauchy e a função $u_{2}$ 
obtida com eles. Note também que a condição de $f$ ser holomórfica na vizinhança de $x=0$ tem sido usada para tomar sua derivada nesse ponto. Isto ocorre também nos seguintes termos na seqüência da equação (63), que são obtidos por derivação sucessiva da equação (61) em $x=0$ : o resultado será sempre que a função $u_{h}$ é um polinômio de $u_{0}, u_{1}, \ldots, u_{h-1}$ e suas derivadas, assim como de $f$ e suas derivadas tomadas nos argumentos da equação 65.

Uma vez que conhecemos as funções $u_{h}$, podemos expressá-las como séries de potências na variável $y$ ao redor do valor $y_{0}$ fixo:

$$
u_{h}(y)=\sum_{k} \frac{u_{h k}}{k !}\left(y-y_{0}\right)^{k}
$$

e, então, a solução completa do problema de Cauchy é:

$$
u(x ; y)=\sum_{h, k} \frac{u_{h k}}{h ! k !} x^{h}\left(y-y_{0}\right)^{k},
$$

com todos os coeficientes $u_{h k}$ determinados pelos dados de Cauchy. Como os coeficientes são únicos, podemos estabelecer: O problema de Cauchy não admite mais de uma solução holomórfica, representada pela série da equação (68). Essa é a unicidade da solução. Sua existência depende do fato da série da equação 68 ser convergente para $|x|$ e $\left|y-y_{0}\right|$ limitados por certos valores apropriados. Mas, em síntese, o que temos aprendido é que uma equação diferencial parcial de segunda ordem possui problema de Cauchy bem definido se especificarmos a função e sua primeira derivada em relação a uma de suas variáveis numa superfície em que dita variável adota um valor fixo, e que, ademais, se a solução existe, então ela é única.

Problema de Cauchy-Kovalevskaya para a equação de Klein-Gordon-Fock. Apliquemos então este conhecimento à equação de Klein-Gordon-Fock [equação (56)]. Como estamos interessados na evolução temporal da solução, escolheremos a variável $t$ para enunciar o problema de Cauchy - notando que essa escolha determina já o uso da dinâmica instantânea-: Seja $u(x)=$ $u(t ; \boldsymbol{x})$ uma função que satisfaz à equação de segunda ordem:

$$
\frac{\partial^{2} u}{\partial t^{2}}(x)=\left(\nabla^{2}-m^{2}\right) u(x),
$$

sujeita aos seguintes dados de Cauchy na superfície espacial tridimensional $t=0$ :

$$
u(0 ; \boldsymbol{x})=u_{0}(\boldsymbol{x}) \quad \text { e } \quad \partial_{t} u(0 ; \boldsymbol{x})=u_{1}(\boldsymbol{x}) .
$$

Logo, vemos que a equação (69) tem a forma da equação 61) e, portanto, a tese do teorema de CauchyKovalevskaya é aplicável. Para solucionar tal equação passamos ao espaço dos momentos por meio da transformação de Fourier aplicada à função $u$. Substituíndo-a na equação 69 vemos que ela tem de ter a forma:

$$
\begin{aligned}
u(x)= & (2 \pi)^{-2} \int d^{4} p \delta\left(p^{2}-m^{2}\right) u(p) e^{-i p x} \\
= & (2 \pi)^{-2} \int \frac{d^{4} p}{\left|2 p_{0}\right|}\left[\delta\left(p_{0}-\omega_{p}\right)+\delta\left(p_{0}+\omega_{p}\right)\right] \\
& \times u(p) e^{-i p x},
\end{aligned}
$$

com: $\omega_{p} \equiv \sqrt{\boldsymbol{p}^{2}+m^{2}}$-pode-se reconhecer aqui a forma do gerador $P_{0}$ da equação 32 -. Integrando na variável $p_{0}$ com o uso das distribuições delta de Dirac, obtemos a separação em partes de freqüência positiva e freqüência negativa:

$$
\begin{aligned}
u(x)= & (2 \pi)^{-2} \int \frac{d^{3} \boldsymbol{p}}{2 \omega_{p}}\left[\left.u_{+}(\boldsymbol{p}) e^{-i p x}\right|_{p_{0}=\omega_{p}}\right. \\
& \left.+\left.u_{-}(\boldsymbol{p}) e^{-i p x}\right|_{p_{0}=-\omega_{p}}\right] .
\end{aligned}
$$

Coloquemos aqui os dados de Cauchy da equação 70 . Primeiramente, como $u_{0}(\boldsymbol{x})=u(0 ; \boldsymbol{x})$ :

$$
u_{0}(\boldsymbol{x})=(2 \pi)^{-2} \int \frac{d^{3} \boldsymbol{p}}{2 \omega_{p}}\left[u_{+}(\boldsymbol{p})+u_{-}(\boldsymbol{p})\right] e^{i \boldsymbol{p} \cdot \boldsymbol{x}} .
$$

Mas podemos escrever:

$$
\begin{aligned}
u_{0}(\boldsymbol{x}) & =\int d^{3} \boldsymbol{y} u_{0}(\boldsymbol{y}) \delta(\boldsymbol{x}-\boldsymbol{y}) \\
& =(2 \pi)^{-3} \int d^{3} \boldsymbol{p}\left(\int d^{3} \boldsymbol{y} u_{0}(\boldsymbol{y}) e^{-i \boldsymbol{p} \cdot \boldsymbol{y}}\right) e^{i \boldsymbol{p} \cdot \boldsymbol{x}},
\end{aligned}
$$

a partir do qual obtemos, via uma comparação das equações (74) e (75) e a independência linear das funções exponenciais $e^{i \boldsymbol{p} \cdot \boldsymbol{x}}$, que:

$$
u_{+}(\boldsymbol{p})+u_{-}(\boldsymbol{p})=2 \omega_{p}(2 \pi)^{-1} \int d^{3} \boldsymbol{y} u_{0}(\boldsymbol{y}) e^{-i \boldsymbol{p} \cdot \boldsymbol{y}} .
$$

Usaremos agora os dados de Cauchy referidos à derivada de $u$. Como $u_{1}(\boldsymbol{x})=\partial_{t} u(0 ; \boldsymbol{x})$ :

$$
u_{1}(\boldsymbol{x})=(2 \pi)^{-2} \int \frac{d^{3} \boldsymbol{p}}{2 \omega_{p}}\left(-i \omega_{p}\right)\left[u_{+}(\boldsymbol{p})-u_{-}(\boldsymbol{p})\right] e^{i \boldsymbol{p} \cdot \boldsymbol{x}},
$$

e, novamente reescrevendo o lado esquerdo com ajuda da distribuição delta de Dirac tridimensional e comparando com a equação (77), encontramos que:

$$
u_{+}(\boldsymbol{p})-u_{-}(\boldsymbol{p})=2 i(2 \pi)^{-1} \int d^{3} \boldsymbol{y} u_{1}(\boldsymbol{y}) e^{-i \boldsymbol{p} \cdot \boldsymbol{y}} .
$$

As equações 76 e 78 nos permitem encontrar explicitamente as funções $u_{ \pm}(\boldsymbol{p})$ em dependência com os dados de Cauchy $u_{0}$ e $u_{1}$ :

$$
u_{ \pm}(\boldsymbol{p})=(2 \pi)^{-1} \int d^{3} \boldsymbol{y}\left[\omega_{p} u_{0}(\boldsymbol{y}) \pm i u_{1}(\boldsymbol{y})\right] e^{-i \boldsymbol{p} \cdot \boldsymbol{y}} .
$$


Substituíndo-as na equação 73 e completando $\boldsymbol{p} \cdot \boldsymbol{y}=$ $\left.p y\right|_{y^{0}=0}$, o que serve para escrever a integração com medida $d^{3} \boldsymbol{y}$ explícitamente na superfície $y^{0}=0$ em que são fornecidos os dados de Cauchy:

$$
\begin{aligned}
u(x)= & (2 \pi)^{-3} \int d^{4} p \delta\left(p^{2}-m^{2}\right) \int_{y^{0}=0} d^{3} \boldsymbol{y} \\
& \times\left[\omega_{p} u_{0}(\boldsymbol{y})+i \operatorname{sgn}\left(p_{0}\right) u_{1}(\boldsymbol{y})\right] e^{-i p(x-y)} .
\end{aligned}
$$

É possível escrever:

$$
\omega_{p} e^{-i p(x-y)}=i \operatorname{sgn}\left(p_{0}\right) \partial_{0}^{x} e^{-i p(x-y)},
$$

e assim:

$$
u(x)=\int_{y^{0}=0} d^{3} \boldsymbol{y}\left[u_{0}(\boldsymbol{y}) \partial_{0}^{x} D(x-y)+D(x-y) u_{1}(\boldsymbol{y})\right]
$$

com a definição da «distribuição de Jordan-Pauli»:

$$
D(x):=i(2 \pi)^{-3} \int d^{4} p \delta\left(p^{2}-m^{2}\right) \operatorname{sgn}\left(p_{0}\right) e^{-i p x} .
$$

Esta distribuição tem duas propriedades imediatas: Por causa da distribuição delta de Dirac que aparece em seu integrando, ela satisfaz a equação de Klein-Gordon-Fock com massa $m$, isto é, a mesma equação diferencial do campo $u$ :

$$
\left(\square+m^{2}\right) D(x)=0 .
$$

Também, de sua forma explícita dada na equação (83):

$$
D(x)=-D(-x)
$$

Fazendo uso, precisamente, desta última propriedade, podemos escrever a equação 82 com a derivada que age sobre $D(x-y)$ não sendo já na variável $x^{0}$, mas na variável $y^{0}$ :

$$
u(x)=\int_{y^{0}=0} d^{3} \boldsymbol{y}\left[u_{0}(\boldsymbol{y}) \partial_{0}^{y} D(y-x)-D(y-x) u_{1}(\boldsymbol{y})\right] .
$$

Essa mudança é útil porque, lembremos: $u_{1}(\boldsymbol{y})=$ $\partial_{0}^{y} u(0 ; \boldsymbol{y})$; mas o fato de ser $y^{0}=0$ já está indicado na integral e, então, podemos escrever simplesmente, dentro dela, $u_{1}(\boldsymbol{y})=\partial_{0}^{y} u(y)$ e $u_{0}(\boldsymbol{y})=u(y)$. Assim:

$$
u(x)=\int_{y^{0}=0} d^{3} \boldsymbol{y} u(y) \overleftrightarrow{\partial}_{0}^{y} D(y-x)
$$

Aqui, o símbolo $f \overleftrightarrow{\partial} g$ significa $f \partial g-\partial f g$. Usando mais uma vez a equação (85), a equação 87) é igual a:

$$
u(x)=\int_{y^{0}=0} d^{3} \boldsymbol{y} D(x-y) \overleftrightarrow{\partial}{ }_{0}^{y} u(y)
$$

Essa é a solução da equação de Klein-Gordon-Fock com dados de Cauchy na superfície $y^{0}=0$, que, existindo, é única, como demonstrado na construção anterior. A causalidade da qual falávamos se evidencia na expressão -os detalhes do cálculo são mostrados, por exemplo, na Ref. [53]-:

$$
D(x)=\frac{1}{2 \pi} \operatorname{sgn}\left(x^{0}\right)\left[\delta(x)-\Theta\left(x^{2}\right) \frac{m}{2 \sqrt{x^{2}}} J_{1}\left(m \sqrt{x^{2}}\right)\right],
$$

o que implica que:

$$
\operatorname{supp}(D(x))=V^{+}(0) \cup V^{-}(0),
$$

com:

$$
V^{+}(x):=\left\{y \in \mathbb{M} \mid(y-x)^{2} \geq 0 \wedge y^{0} \geq x^{0}\right\}
$$

o «cone de luz futuro em relação ao ponto $x »$, e

$$
V^{-}(x):=\left\{y \in \mathbb{M} \mid(y-x)^{2} \geq 0 \wedge y^{0} \leq x^{0}\right\}
$$

o «cone de luz passado em relação ao ponto $x »$. Para terminar de introduzir a nomenclatura, digamos que a união $V^{+}(x) \cup V^{-}(x)$ é o «cone de luz com vértice no ponto $x \gg$. Isto é, $D(x)$ tem suporte causal em relação à origem do sistema de coordenadas. Vejamos o que isto quer dizer à luz da equação (87): Da equação (90) temos que, transladando a origem até o ponto $x$ em que queremos conhecer o valor do campo $u, D(y-x)$ tem suporte no cone de luz com vértice em $x$, isto é, $u(x)$ não é afetado pelos valores que no passado o campo tomou fora do cone de luz de $x$, nem afetará os valores que o campo tomará fora do cone de luz de $x$ no futuro.

\subsection{Problema de Goursat}

Consideremos agora que: nossa solução, embora satisfatória, não é tão geral como gostaríamos, pois precisa do conhecimento dos dados iniciais numa superfície de tempo constante, o que obriga a escolha da forma dinâmica instantânea. Mas, o que aconteceria se os dados fossem conhecidos numa superfície diferente? Voltemos ao problema de valores iniciais como enunciado na equação (57), e suponhamos que o espaço $m$-dimensional seja submetido à transformação de coordenadas:

$$
X_{1}=G_{1}\left(x_{1} ; \cdots ; x_{m}\right), \cdots, X_{m}=G_{m}\left(x_{1} ; \cdots ; x_{m}\right),
$$

de tal forma que $X_{m}=G_{m}\left(x_{1} ; \cdots ; x_{m}\right)=0$ seja a superfície em que são conhecidos os dados iniciais. A equação diferencial é substituída por uma análoga para as novas coordenadas, com $u\left(x_{1} ; \cdots ; x_{m}\right)=U\left(G_{1}\right.$ $\left.\left(x_{1} ; \cdots ; x_{m}\right) ; \cdots ; G_{m}\left(x_{1} ; \cdots ; x_{m}\right)\right)$ :

$$
\Phi\left(U ; X_{i} ; \frac{\partial U}{\partial X_{i}} ; \frac{\partial^{2} U}{\partial X_{i}^{2}} ; \frac{\partial^{2} U}{\partial X_{i} \partial X_{k}}\right)=0 .
$$

Esta simples transformação de coordenadas, poderia parecer, não tem maiores implicações na teoria, pois 
parece que podemos simplesmente aplicar o teorema de Cauchy-Kovalevskaya à nova equação diferencial. Porém, suponhamos que a equação (59) seja linear, e que tem portanto a seguinte forma geral:

$$
\sum_{i, k} A_{i k} \frac{\partial^{2} u}{\partial x_{i} \partial x_{k}}+\sum_{i} B_{i} \frac{\partial u}{\partial x_{i}}+C u=f,
$$

com $A_{i k}=A_{k i}, B_{i}, C$ e $f$ funções das variáveis $x_{i}$. Suponhamos agora que as transformações da equação (93) sejam feitas. Então usando a regra da cadeia obtemos que a equação 95 é, em relação às variáveis $X_{i}$ :

$$
\begin{aligned}
& \sum_{j, l}\left(\sum_{i, k} A_{i k} \frac{\partial G_{j}}{\partial x_{i}} \frac{\partial G_{l}}{\partial x_{k}}\right) \frac{\partial^{2} U}{\partial X_{j} \partial X_{l}} \\
& +\sum_{l}\left(\sum_{i} B_{i} \frac{\partial G_{l}}{\partial x_{i}}\right) \frac{\partial U}{\partial X_{l}}+C U=f .
\end{aligned}
$$

Como temos observado, o teorema de CauchyKovalevskaya tem por hipótese o cumprimento da equação 60 , isto é, ele será aplicável só se $\partial^{2} U / \partial X_{m}^{2}$ aparece na equação 96 . Isto coloca em primeiro plano a «forma característica» A definida com 5 .

$$
\mathbf{A}\left(\gamma_{1} ; \cdots ; \gamma_{m}\right):=\sum_{i, k} \gamma_{i} A_{i k} \gamma_{k}
$$

Da equação 96 vemos que a hipótese do teorema de Cauchy-Kovalevskaya não se satisfaz se:

$$
\sum_{i, k} A_{i k} \frac{\partial G_{m}}{\partial x_{i}} \frac{\partial G_{m}}{\partial x_{k}}=0
$$

Este é o único caso em que a generalização para superfícies iniciais quaisquer não pode ser realizada trivialmente. As superfícies $G_{m}\left(x_{1} ; \cdots ; x_{m}\right)=0$ para as quais isto acontece recebem o nome de «superfícies características» da equação diferencial. O problema dos valores iniciais em que a superfície inicial é uma superfície característica é conhecido como «problema de Goursat» [54, e o desenvolveremos a seguir.

Seja a seguinte equação diferencial em que a segunda derivada em relação à variável $x_{m}$ não pode ser isolada, pois não aparece; ou seja, a equação diferencial escrita

\footnotetext{
${ }^{5}$ Recordamos que a forma característica define o tipo de equação diferencial parcial da segunda ordem [54]: (i) Se ela contém $m$ autovalores diferentes não nulos do mesmo sinal, como acontece, por exemplo, com a equação de Laplace $\nabla^{2} \varphi=0$, ela se chama «elíptica»; (ii) se tem menos de $m$ autovalores não nulos, como acontece com a equação de Schrödinger $\partial_{t} \psi+\nabla^{2} \psi=0$, ela se chama «parabólica»; (iii) se tem $m$ autovalores não nulos, não todos do mesmo sinal, como acontece com a equação de Klein-Gordon-Fock [equação [69], então a equação diferencial denomina-se «hiperbólica». Particularmente, se na equação hiperbólica a forma característica tem todos os autovalores do mesmo sinal, exceto um, então ela se chama «hiperbólica normal»; é a esse tipo que pertence a equação de Klein-Gordon-Fock.
}

em termos de coordenadas tais que a superfície $x_{m}=0$ é uma superfície característica $\left(A_{m m}=0\right)$ :

$$
\sum_{i \neq k} A_{i k} \frac{\partial^{2} u}{\partial x_{i} \partial x_{k}}+\sum_{i=1}^{m-1} A_{i i} \frac{\partial^{2} u}{\partial x_{i}^{2}}+\sum_{i} B_{i} \frac{\partial u}{\partial x_{i}}+c u=f .
$$

Nosso primeiro propósito será ver se os dados iniciais que são suficientes no problema fora de superfícies características são também suficientes nesse caso. Suponhamos, pois, que sejam conhecidos:

$$
\begin{aligned}
& u\left(x_{1} ; \cdots ; x_{m-1} ; 0\right)=u_{0}\left(x_{1} ; \cdots ; x_{m-1}\right) ; \\
& \frac{\partial u}{\partial x_{m}}\left(x_{1} ; \cdots ; x_{m-1} ; 0\right)=u_{1}\left(x_{1} ; \cdots ; x_{m-1}\right) .
\end{aligned}
$$

Façamos, como anteriormente, a expansão ao redor de $x_{m}=0$ :

$$
\begin{aligned}
& u=u_{0}+u_{1} x_{m}+\cdots+\frac{u_{h}}{h !} x_{m}^{h}+\cdots ; \\
& u_{h}=\left.u_{h}\left(x_{1} ; \cdots ; x_{m}\right) \equiv \frac{\partial^{h} u}{\partial x_{m}^{h}}\right|_{x_{m}=0} .
\end{aligned}
$$

Colocando essa expansão na equação 99 , então avaliando em $x_{m}=0$, obtemos já não uma expressão para $u_{2}$ como outrora obtivemos, mas uma equação diferencial para $u_{1}$ :

$$
2 \sum_{i \neq m} A_{i m} \frac{\partial u_{1}}{\partial x_{i}}+B_{m} u_{1}+H_{1}=0,
$$

com $H_{1}$ dependendo somente de $u_{0}$ e suas derivadas:

$$
\begin{aligned}
H_{1}= & \sum_{i \neq m, k \neq m, i \neq k} A_{i k} \frac{\partial^{2} u_{0}}{\partial x_{i} \partial x_{k}}+\sum_{i \neq m} A_{i i} \frac{\partial^{2} u_{0}}{\partial x_{i}^{2}} \\
& +\sum_{i \neq m} B_{i} \frac{\partial u_{0}}{\partial x_{i}}+C u_{0}-f .
\end{aligned}
$$

Vemos, pois, que se $u_{1}$ não for dado de forma a satisfazer essa equação diferencial, então o problema de valores iniciais com os dados iniciais da equação 100 não tem solução. Portanto, precisamos fornecer outros dados iniciais, a saber, os necessários para que as equações diferenciais que se obtêm para as funções $u_{h}$ possam ser univocamente solucionadas.

A equação 102 é uma equação diferencial parcial de primeira ordem para $u_{1}$. Vejamos se também as equações para os outros $u_{h}$ são desse tipo. Derivando a equação (99) $h-1$ vezes em relação a $x_{m}$ e então tomando $x_{m}=0$, obtemos a equação diferencial para $u_{h}$ :

$$
2 \sum_{i \neq m} A_{i m} \frac{\partial u_{h}}{\partial x_{i}}+B_{m} u_{h}+H_{h}=0
$$

com:

$$
\begin{aligned}
H_{h}= & \sum_{i \neq m, k \neq m, i \neq k} A_{i k} \frac{\partial^{2} u_{h-1}}{\partial x_{i} \partial x_{k}}+\sum_{i \neq m} A_{i i} \frac{\partial^{2} u_{h-1}}{\partial x_{i}^{2}} \\
& +\sum_{i \neq m} B_{i} \frac{\partial u_{h-1}}{\partial x_{i}}+C u_{h-1}-\frac{\partial^{h-1} f}{\partial x_{m}^{h-1}} .
\end{aligned}
$$


Observamos que todas as equações diferenciais que se obtêm para as funções $u_{h}$ são de primeira ordem. Para solucioná-las, estudemos o método das características para esse tipo de equações. Para simplificar a exposição, além disso, consideremos o caso de duas variáveis e suponhamos que queremos resolver a equação:

$$
A(x ; y) \frac{\partial u}{\partial x}+B(x ; y) \frac{\partial u}{\partial y}-C(x ; y ; u)=0 .
$$

As equações 104 são todas desse tipo, com a particularidade de ser $A(x ; y)=0$, o que colocaremos no final. Solucionar a equação 106 significa determinar uma solução $u=z(x ; y)$; essa superfície solução tem vetor normal $\boldsymbol{n}=(\partial z / \partial x ; \partial z / \partial y ;-1)$; portanto, definindo o campo vetorial $\boldsymbol{F}=(A ; B ; C)$, a equação 106 pode ser escrita como:

$$
\boldsymbol{F} \cdot \boldsymbol{n}=0 \text {. }
$$

Ou seja, $\boldsymbol{F}$ é tangente à superfície solução $u=z(x ; y)$, tendo como consequência que esta é constituída por curvas integrais daquela; tais curvas integrais são chamadas «curvas características». Isto leva imediatamente à seguinte estratégia de solução: Sejam dados os valores de $u(x ; y)$ numa curva $\gamma$ no plano $x-y$, cujo uso permite estabelecer a curva $\Gamma$ sobre a superfície $z(x ; y)$ que lhe corresponde. Então a partir de cada ponto de $\Gamma$ pode ser gerada uma curva integral de $\boldsymbol{F}$, construindo assim a superfície $z(x ; y)$. Levando a ideia à prática, podemos parametrizar, para $a$ em algum domínio de variação:

$$
\Gamma(a)=\left(x=x_{0}(a) ; y=y_{0}(a) ; z=z_{0}(a)\right) .
$$

Determinar para cada valor de $a$ uma curva integral de $\boldsymbol{F}$ que passe por $\Gamma(a)$ significa resolver, para cada valor de $a$, o «sistema de equações diferenciais de LagrangeCharpit»: Para um parâmetro $s$ :

$$
\begin{aligned}
& \frac{d x}{d s}=A(x ; y) ; \quad x(0)=x_{0}(a), \\
& \frac{d y}{d s}=B(x ; y) ; \quad y(0)=y_{0}(a), \\
& \frac{d z}{d s}=C(x ; y ; z) ; \quad z(0)=z_{0}(a) .
\end{aligned}
$$

As soluções desse sistema,

$$
x=X(a ; s), \quad y=Y(a ; s), \quad z=Z(a ; s),
$$

são já uma representação paramétrica da superfície solução. Para obtê-la na forma $u=z(x ; y)$, precisaremos inverter:

$$
a=\Lambda(x ; y) \quad \text { e } \quad s=S(x ; y)
$$

com o qual a solução buscada será:

$$
u(x ; y)=Z(\Lambda(x ; y) ; S(x ; y)) .
$$

Vale ressaltar que para que possa ser gerada a superfície solução a partir da curva $\Gamma$ por ação do campo vetorial
$\boldsymbol{F}$, é preciso que $\Gamma$ não seja já uma curva característica uma curva integral de $\boldsymbol{F}$-, pois então ela seria invariante pela ação de $\boldsymbol{F}$. Vejamos como isto funciona em nosso caso: As equações 104 dão lugar às equações de Lagrange-Charpit com o lado direito da equação 109 nulo:

$$
\frac{d x}{d s}=0 ; \quad x(0)=x_{0}(a)
$$

Isto é, $x$ é independente do parâmetro $s$. Se os dados iniciais fossem dados numa linha de $x=$ constante, então $x$ seria também independente de $a$; logo não poderíamos obter a solução do sistema mais que para um valor constante de $x$. Concluimos: Os dados iniciais que são precisos para solucionar univocamente as equações (104) não podem ser dados na superfície $x_{m}=0$, mas têm de ser dados em uma outra superfície. Por exemplo, se os dados iniciais estão na linha de $y$ constante:

$$
\Gamma=\left(x=x_{0}(a) ; y=y_{0} ; z=z_{0}(a)\right),
$$

com $y_{0}$ independente de $a$. A equação 115 tem solução: $x=x_{0}(a)$. Substituíndo na equação (110), como $x$ é independente de $s$, a integração se torna elementar:

$$
s=\int_{y_{0}}^{y} \frac{d y}{B\left(x_{0}(a) ; y\right)} .
$$

Finalmente, a equação 111 será:

$$
\frac{d z}{d s}=C\left(x_{0}(a) ; y(a ; s) ; z\right) ; \quad z(0)=z_{0}(a),
$$

que é uma equação bem definida e com condições iniciais, portanto solúvel e com solução única.

A análise recém realizada demonstra que as equações (104) que determinam as funções $u_{h}$ poderão ser resolvidas univocamente se são fornecidos dados iniciais para $u$ numa superfície, por exemplo, com $x_{k}=$ constante, $k \neq m$. Conclui-se que, quando o problema de valores iniciais está definido sobre uma superfície característica da equação diferencial, os dados iniciais não podem ser o valor da função e sua primeira derivada normal à superfície característica, pois em tal caso o problema pode não ter solução ou ter infinitas soluções. Em seu lugar, devem ser dados: o valor da função na superfície característica e o valor da função em uma superfície diferente dela.

\subsection{Superfícies características da equação de Klein-Gordon-Fock}

Uma vez que temos visto que o problema de valores iniciais pode ser bem definido sobre superfícies características, apliquemos o conhecimento adquirido para a equação de Klein-Gordon-Fock com que iniciamos essa discusão [equação (56)]:

$$
\frac{\partial^{2} u}{\partial t^{2}}-\frac{\partial^{2} u}{\partial x^{2}}-\frac{\partial^{2} u}{\partial y^{2}}-\frac{\partial^{2} u}{\partial z^{2}}+m^{2} u=0
$$


Comparando com a equação (95), reconhecemos que para essa equação sã ${ }^{6}$

$$
A_{\mu \nu}=\widetilde{\eta}_{\mu \nu}, \quad B_{\mu}=0, \quad C=m^{2} \quad \text { e } \quad f=0 .
$$

Aqui, $\widetilde{\eta}_{\mu \nu}$ são as componentes do tensor métrico do espaço-tempo de Minkowski: $\left(\widetilde{\eta}_{\mu \nu}\right)=$ $\operatorname{diag}(+1 ;-1 ;-1 ;-1)$. A forma característica [vide a equação (97)] é aqui $\widetilde{\eta}_{\mu \nu} \gamma^{\mu} \gamma^{\nu}$, que se anula para $\gamma^{\mu}$ um vetor tipo-luz:

$$
\left(\gamma^{0}\right)^{2}=\gamma^{2}
$$

Assim sendo, a transformação de coordenadas $X_{\mu}=$ $G_{\mu}(t ; x ; y ; z)$ será tal que $X_{0}=$ constante é uma superfície característica se [vide a equação (98)] a função $G_{0}$ satisfaz à equação diferencial:

$$
\left(\frac{\partial G_{0}}{\partial t}\right)^{2}-\left(\frac{\partial G_{0}}{\partial x}\right)^{2}-\left(\frac{\partial G_{0}}{\partial y}\right)^{2}-\left(\frac{\partial G_{0}}{\partial z}\right)^{2}=0
$$

cuja solução é:

$$
G_{0}=a t+b x+c y+d z ; \quad a= \pm \sqrt{b^{2}+c^{2}+d^{2}} .
$$

Isto é, as superfícies características da equação de KleinGordon-Fock são as superfícies $X_{0}=0$, com:

$$
X_{0} \sim t \pm \frac{b x+c y+d z}{\sqrt{b^{2}+c^{2}+d^{2}}}
$$

ou seja, planos nulos, com as constantes $b, c$ e $d$, assim como o fator de proporcionalidade com a coordenada $X_{0}$, podendo ser arbitráriamente escolhidas. Façamos uma escolha:

Definição: Sejam $\left(x^{0} ; x^{1} ; x^{2} ; x^{3}\right)$ coordenadas cartesianas no espaço-tempo de Minkowski tais que o tensor métrico adota sua forma diagonal normalizada $\widetilde{\eta}_{\mu \nu}$. $O$ conjunto de coordenadas $\left(x^{+} ; x^{1} ; x^{2} ; x^{-}\right)$, com:

$$
x^{+}:=\frac{1}{\sqrt{2}}\left(x^{0}+x^{3}\right), \quad x^{-}:=\frac{1}{\sqrt{2}}\left(x^{0}-x^{3}\right),
$$

é chamado conjunto de coordenadas do plano nulo.

A eleição da superfície característica em que os dados iniciais sejam dados, quer $x^{+}=0$, quer $x^{-}=0$, é um assunto puramente convencional, mas importante por ser, implícitamente, uma eleição do parâmetro de evolução dos campos, que doravante chamaremos «tempo». Escolhemos $x^{+}$como coordenada temporal; a seguinte nomenclatura é usada: $x^{-}$é a coordenada «longitudinal», e $x^{\perp}=\left(x^{1} ; x^{2}\right)$ são as coordenadas «transversais». As coordenadas cartesianas $\left(x^{0} ; x^{1} ; x^{2} ; x^{3}\right)$ passarão a ser chamadas «coordenadas instantâneas», por razões óbvias. O fator de proporcionalidade escolhido na definição de $x^{ \pm}$na equação 125 tem a virtude de que,

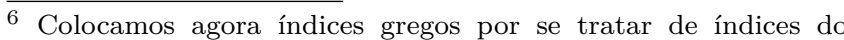
espaço-tempo.
}

com ele, o determinante jacobiano da transformação de coordenadas é igual à unidade, de forma que o elemento de volume não muda:

$$
d^{4} x=d x^{+} d x^{-} d^{2} x^{\perp} .
$$

Também, chamando $\eta_{a b}$ a métrica nas coordenadas do plano nulo, ela tem a seguinte representação matricial:

$$
\left[\eta_{a b}\right]=\left(\begin{array}{cccc}
0 & 0 & 0 & 1 \\
0 & -1 & 0 & 0 \\
0 & 0 & -1 & 0 \\
1 & 0 & 0 & 0
\end{array}\right)=\left[\eta^{a b}\right]
$$

Pelo proferido, concluímos que o problema de Goursat da equação de Klein-Gordon-Fock é o problema dos valores iniciais na dinâmica da frente de luz. E mais ainda: Essa é a única exceção à aplicabilidade do teorema de Cauchy-Kovalevskaya: É a única forma dinâmica na qual o problema dos valores iniciais não pode ser obtido por deformação da superfície inicial em alguma outra forma dinâmica.

Passemos a estabelecer a solução do problema de Goursat da equação de Klein-Gordon-Fock. Para isso, lembremos da equação 88): Em coordenadas instantâneas:

$$
u(x)=\int_{y^{0}=0} d^{3} \boldsymbol{y} D(x-y) \overleftrightarrow{\partial}_{0}^{y} u(y)
$$

Denotemos a versão tetra-vetorial do integrando por $N^{\mu}(x ; y)$ :

$$
N^{\mu}(x ; y)=D(x-y) \overleftrightarrow{\partial}_{y}^{\mu} u(y)
$$

Tomando a divergência em relação à variável $y$, vemos que:

$$
\partial_{\mu}^{y} N^{\mu}(x ; y)=D(x-y) \square_{y} u(y)-\square_{y} D(x-y) u(y)=0,
$$

pois tanto $u$ como $D$ satisfazem à equação de KleinGordon-Fock de massa $m$ [vide a equação (84)]. Portanto, sendo de divergência nula, podemos usar o teorema de Gauss no volume $\Omega$ limitado pelo sólido $A-B-C-D$ na Fig. 4-compare-se essa figura com a 3 , em que o ponto $P$, vértice do cone de luz, representa o ponto $x$ em que desejamos conhecer o valor do campo u. Então teremos que:

$$
\begin{aligned}
0= & \int_{\Omega} d^{4} y \partial_{\mu}^{y} N^{\mu}(x ; y)=\int_{\partial \Omega} d \sigma_{\mu}(y) N^{\mu}(x ; y) \\
= & \left(\int_{A-B}+\int_{B-C}+\int_{C-D}+\int_{D-A}\right) \\
& \times D(x-y) \overleftrightarrow{\partial}_{y}^{\mu} u(y) d \sigma_{\mu}(y) .
\end{aligned}
$$

Porém, $B-C$ está fora do cone de luz do ponto $P$, e a integral nessa superfície se anula, pois $D(x-y)$ tem suporte causal. Assim sendo, identificando a integral sobre 


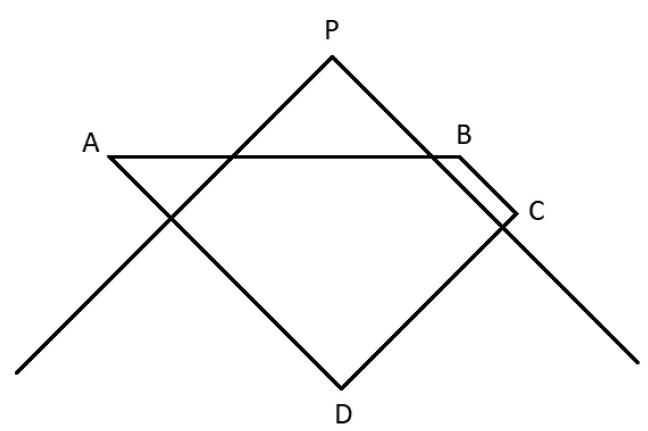

Figura 4: Volume de integração para a obtenção da solução do problema de Goursat da equação de Klein-Gordon-Fock.

$A-B$ como a solução da equação 128 e reconhecendo que os vetores normais às superfícies $C-D$ e $D-A$ são, respectivamente, nas coordenadas do plano nulo, $(0 ; 0 ; 0 ;-1)$ e $(-1 ; 0 ; 0 ; 0)$, obteremos que:

$$
\begin{aligned}
u(x)= & \int_{D-A} D(x-y) \overleftrightarrow{\partial}_{-}^{y} u(y) d^{2} y^{\perp} d y^{-} \\
& +\int_{C-D} D(x-y) \overleftrightarrow{\partial}_{+}^{y} u(y) d^{2} y^{\perp} d y^{+}
\end{aligned}
$$

visto que a superfície $D-A$ é de $x^{+}=$constante e a $C-D$ é de $x^{-}=$constante. Isto é concordante com nossos estudos prévios: A solução ao problema de valores iniciais é única e bem definida se os dados iniciais são o valor do campo na superfície característica e o valor dele em uma superfície diferente da anterior. Em outras palavras, temos substituído o valor de $u$ e $\partial_{0} u$ em um plano tipo-espaço pelo conhecimento de $u$ em dois planos nulos. Em particular, podemos fazer o limite em que a superfície $C-D$ se encontra em $x^{-} \rightarrow-\infty$, e os dados iniciais nessa superfície são substituídos pela «condição assintótica» [14, 15]:

$$
\lim _{x^{-} \rightarrow-\infty} u(x)=0 .
$$

Em tal caso, a solução da equação 132 se simplifica a:

$$
u(x)=\int_{y^{+}=y_{0}^{+}} D(x-y) \overleftrightarrow{\partial_{-}^{y}} u(y) d^{2} y^{\perp} d y^{-}
$$

que precisa do conhecimento só do valor do campo na superfície isocrônica $x^{+}=x_{0}^{+}$.

\section{Coordenadas invariantes do plano nulo}

Como temos estabelecido, a relação entre uma forma dinâmica e outra vai além de uma simples transformação de coordenadas:7 A verdadeira diferença fundamental é

\footnotetext{
7 É claro, uma transformação de coordenadas não oferece nenhuma diferença importante, pois as equações do movimento na teoria da relatividade tem caráter tensorial e são, portanto, covariantes frente a transformações gerais de coordenadas.
}

que com essa mudança temos um novo conjunto de dados iniciais, que evoluem causalmente de uma superfície para outra segundo os geradores dinâmicos da forma particular.

Assim, é possível usar, em qualquer forma dinâmica, qualquer conjunto de coordenadas. Por exemplo, é perfeitamente possível usar coordenadas no plano nulo e ainda assim estar na dinâmica instantânea, se os dados iniciais fossem dados numa superfície de $x^{0}$ constante. Isto porque, já vimos na seção 2 uma transformação das coordenadas não implica uma transformação da superfície inicial. Por isso, para recuperar a covariância da teoria e assim explicitar que o sistema de coordenadas nada tem a ver com a forma dinâmica, Rohrlich [14, 15] introduziu um conjunto de campos vetoriais, tais que seja sempre possível levar as quantidades covariantes à forma «própria» do plano nulo. Consideremos um sistema inercial de referência; ele define sua origem $O$ e seus eixos cartesianos instantâneos segundo a escolha de quatro campos vetoriais $\widetilde{e}^{(0)}, \widetilde{e}^{(1)}, \widetilde{e}^{(2)}$ e $\widetilde{e}^{(3)}$, tais que as coordenadas cartesianas instantâneas de um ponto $P$ qualquer são obtidas por projeçã $q^{8}$

$$
x^{(a)}(P)=: \int_{O}^{P} \widetilde{e}_{\mu}^{(a)} d x^{\mu} ;(a)=(0),(1),(2),(3),
$$

expressão esta que é invariante frente a qualquer substituição das coordenadas. Portanto, uma transformação de coordenadas muda $x^{\mu}$, e, claro, as componentes $\widetilde{e}^{(a)}{ }_{\mu}$, mas o campo vetorial $\widetilde{e}^{(a)}$, assim como as coordenadas $x^{(a)}$, se mantêm as mesmas. Uma transformação de sistema de referência muda os campos vetoriais $\widetilde{e}^{(a)}$ e, em consequência, as coordenadas $x^{(a)}$. Os campos $\widetilde{e}^{(a)}$ são chamados «campos de tétradas», e as coordenadas $x^{(a)}$, «coordenadas invariantes». Mais em geral, também outras grandezas podem ser projetadas com o campo de tétradas para obter sua forma invariante. Em particular, a métrica $g_{\mu \nu}$ se relaciona com a métrica invariante $\widetilde{\eta}_{(a)(b)}$ por:

$$
g_{\mu \nu}(x) \widetilde{e}_{(a)}^{\mu}(x) \widetilde{e}_{(b)}^{\nu}(x)=\widetilde{\eta}_{(a)(b)},
$$

ou, inversamente:

$$
\widetilde{\eta}_{(a)(b)}(x) \widetilde{e}_{\mu}^{(a)}(x) \widetilde{e}_{\nu}^{(b)}(x)=g_{\mu \nu} .
$$

Agora, como já mencionado (vide a Ref. [55]): «[...] a eleição da base de tétradas depende das simetrias subjacentes do espaço-tempo e é, em boa medida, parte do problema». É claro, quando o autor se refere às «simetrias do espaço-tempo», devemos entender que entre elas estão também as simetrias do problema a se resolver. E como temos insistido em que alguns problemas se

\footnotetext{
8 A forma integral aqui é devida a que, no caso geral de se utilizar coordenadas curvas, o simples produto $\widetilde{e}^{(a)}{ }_{\mu} x^{\mu}$ pode não fornecer a informação desejada. Tal produto será suficiente, por outra parte, se as coordenadas $x^{\mu}$ estão definidas ao longo de eixos retos.
} 
simplificam com o uso da forma dinâmica do plano nulo, algumas vezes será vantajoso escolher a base de tétradas obtida, exigindo que a métrica invariante dessa dinâmica seja igual à métrica nesse conjunto de coordenadas [vide a equação (127]):

$$
\left[\eta_{(a)(b)}\right]=\left(\begin{array}{cccc}
0 & 0 & 0 & 1 \\
0 & -1 & 0 & 0 \\
0 & 0 & -1 & 0 \\
1 & 0 & 0 & 0
\end{array}\right)
$$

Porém, as componentes do tensor métrico $g_{\mu \nu}$ dependem unicamente do sistema de coordenadas, e não da base de tétradas, de forma que, se denotarmos por $e^{(+)}, e^{(1)}, e^{(2)}$ e $e^{(-)}$os campos de tétradas correspondentes ao plano nulo, então:

$$
\begin{aligned}
& \widetilde{e}^{(0)} \widetilde{e}^{(0)}{ }_{\nu}-\widetilde{e}_{\mu}^{(1)} \widetilde{e}_{\nu}^{(1)}-\widetilde{e}_{\mu}^{(2)} \widetilde{e}_{\nu}^{(2)}-\widetilde{e}_{\mu}^{(3)} \widetilde{e}_{\nu}^{(3)}=g_{\mu \nu} \\
& =e^{(+)}{ }_{\mu} e^{(-)}{ }_{\nu}+e^{(-)}{ }_{\mu} e_{\nu}^{(+)}-e^{(1)}{ }_{\mu} e_{\nu}^{(1)}-e_{\mu}^{(2)} e_{\nu}^{(2)},
\end{aligned}
$$

e uma solução $\hat{\epsilon}^{9}$

$$
\begin{aligned}
& e^{(+)}=\frac{1}{\sqrt{2}}\left(\widetilde{e}^{(0)}+\widetilde{e}^{(3)}\right), e^{(-)}=\frac{1}{\sqrt{2}}\left(\widetilde{e}^{(0)}-\widetilde{e}^{(3)}\right), \\
& e^{(1)}=\widetilde{e}^{(1)}, e^{(2)}=\widetilde{e}^{(2)} .
\end{aligned}
$$

E, correspondentemente:

$$
\begin{aligned}
& e_{(+)}=\frac{1}{\sqrt{2}}\left(\widetilde{e}_{(0)}+\widetilde{e}_{(3)}\right), e_{(-)}=\frac{1}{\sqrt{2}}\left(\widetilde{e}_{(0)}-\widetilde{e}_{(3)}\right), \\
& e_{(1)}=\widetilde{e}_{(1)}, e_{(2)}=\widetilde{e}_{(2)} .
\end{aligned}
$$

Os campos de tétradas da dinâmica da frente de luz, por sua definição, apresentam as seguintes propriedades -usaremos doravante $\alpha, \beta=1,2-$ :

$$
\begin{array}{r}
e_{(+)} \cdot e_{(+)}=0=e_{(-)} \cdot e_{(-)}, \quad e_{(+)} \cdot e_{(-)}=1, \\
e_{(\alpha)} \cdot e_{(\beta)}=-\delta_{\alpha \beta}, \quad e_{(+)} \cdot e_{(\alpha)}=0=e_{(-)} \cdot e_{(\alpha)} .
\end{array}
$$

Particularmente, é fácil verificar que quando as tétradas assumem seus valores canônicos, isto é, com componentes $e_{(+)}=(1 ; 0 ; 0 ; 0), e_{(1)}=(0 ; 1 ; 0 ; 0), e_{(2)}=$ $(0 ; 0 ; 1 ; 0)$ e $e_{(-)}=(0 ; 0 ; 0 ; 1)$, então, a métrica $g_{\mu \nu}$ se iguala com a métrica invariante: $\left[g_{\mu \nu}\right]=\left[\eta_{(a)(b)}\right]$ dada na equação 138, o que significa que as coordenadas invariantes coincidem com as coordenadas no plano nulo, como deve ser por consistência.

Sendo o conjunto $\left\{e_{(a)}\right\}(a=+, 1,2,-)$ uma base do espaço-tempo de Minkowski, podemos decompor qualquer vetor A como combinação linear de seus elementos. Escrevemos: $\mathrm{A}=\sum_{(a)} A^{(a)} e_{(a)}$. Multiplicando por um elemento da base dual -base de co-tétradas- $e^{(b)}$ :

$$
e^{(b)} \cdot \mathrm{A}=\sum_{(a)} A^{(a)} e^{(b)} \cdot e_{(a)}=\sum_{(a)} A^{(a)} \delta_{(a)}^{(b)}=A^{(b)} .
$$

\footnotetext{
${ }_{9}$ Essa solução é tomada assim por compatibilidade com a definição das coordenadas no plano nulo dada na equação 125 .
}

Portanto, o vetor A tem componentes invariantes:

$$
A^{(+)}:=\mathrm{A} \cdot e^{(+)}, A^{(\alpha)}:=\mathrm{A} \cdot e^{(\alpha)}, A^{(-)}:=\mathrm{A} \cdot e^{(-)},
$$

e decomposição na base de tétradas:

$$
\mathrm{A}=A^{(+)} e_{(+)}+A^{(\perp)} e_{(\perp)}+A^{(-)} e_{(-)},
$$

com: $A^{(\perp)} e_{(\perp)} \equiv \sum_{\alpha=1}^{2} A^{(\alpha)} e_{(\alpha)}$. O produto escalar de dois vetores $A$ e $B$ pode ser escrito agora em função de suas componentes invariantes no plano nulo:

$$
\mathrm{A} \cdot \mathrm{B}=A^{(+)} B^{(-)}+A^{(-)} B^{(+)}-A^{(\perp)} B^{(\perp)} .
$$

Em particular, o vetor posiçã ${ }^{10} \times$ tem decomposição: $\mathrm{x}=x^{(+)} e_{(+)}+x^{(-)} e_{(-)}+x^{(\perp)} e_{(\perp)}$, e também o operador derivada: $\partial=e_{(+)} \partial_{(-)}+e_{(-)} \partial_{(+)}-e_{(\perp)} \partial_{(\perp)}$, cujas componentes invariantes são dadas pela definição de derivadas direcionais:

$$
\partial_{(+)}=e_{(+)} \cdot \partial=\frac{\partial}{\partial\left(e_{(-)} \cdot x\right)}=\frac{\partial}{\partial x^{(+)}},
$$

e analogamente:

$$
\partial_{(-)}=e_{(-)} \cdot \partial=\frac{\partial}{\partial x^{(-)}}, \quad \partial_{(\perp)}=e_{(\perp)} \cdot \partial=\frac{\partial}{\partial x^{(\perp)}} .
$$

Finalmente, como escolhemos a coordenada $x^{(+)}$como o tempo na dinâmica da frente de luz, o vetor $e_{(+)}$ indicará a direção temporal nessa dinâmica, e $\partial_{(+)}$será a derivada temporal. Cada plano nulo de $x^{(+)}=$constante é gerado pelos três vetores $e_{(\perp)}$ e $e_{(-)}$, e tem elemento de volume que denotaremos por $d^{3} \underline{\boldsymbol{x}}:=d x^{(1)} d x^{(2)} d x^{(-)} \equiv$ $d^{2} x^{(\perp)} d x^{(-)}$.

\section{Formalismo lagrangiano e o primeiro teorema de Nöther}

Uma vez estabelecidos todos os elementos necessários para estudar a física no plano nulo, fixaremos a consideração na teoria de campos clássicos, ou seja, à descrição ondulatória da partícula individual por suas equações de movimento, sem sujeitar ainda suas soluções aos axiomas da teoria quântica -isto é, desconsiderando as exigências da interpretação probabilística, a possibilidade de mudança do número e identidade das partículas, et cetera- .

Uma abordagem, quiçá a mais habitual para o estudo dos campos, é a abordagem lagrangiana. Como ela é bem conhecida e, por outra parte, existem excelentes referências para seu estudo -vide o leitor, por exemplo, as Refs. [53, 59 61]-, nos limitaremos aqui apenas a

\footnotetext{
10 Este é um vetor no espaço-tempo de Minkowski só uma vez que a origem -por exemplo, a posição do observador ou um evento particularmente escolhido- tem sido fixado. Isto é devido ao caráter afim e não simplesmente linear dele. A respeito deste ponto, consultar a Ref. [56].
} 
oferecer um brevíssimo resumo com o intuito de fixar a notação.

Seja $u^{A}(x)(A=1, \cdots, N)$ a componente $A$-ésima de um conjunto de $N$, definidas no espaço-tempo tetradimensional de Minkowski, M. Tais componentes são genéricas, isto é, podem pertencer a campos de diferente natureza. A integral de ação extendida à região espaçotemporal $\Omega$, é definida como:

$$
\mathscr{A}[u]:=\int_{\Omega} d^{4} x \mathscr{L}[u ; \partial u](x),
$$

com $\mathscr{L}$ a densidade lagrangiana de primeira ordem, isto é, que depende das derivadas dos campos de até primeira ordem. Se aplicarmos uma transformação cujos efeitos nas coordenadas e campos são dados, infinitesimalmente, pelas equações:

$$
x^{\prime \mu}=x^{\mu}+\delta x^{\mu}, u^{\prime A}\left(x^{\prime}\right)=u^{A}(x)+\delta u^{A}(x),
$$

então, frente a essa transformação, a ação tem variação:

$$
\begin{aligned}
\delta \mathscr{A}[u] & :=\int_{\Omega^{\prime}} d^{4} x^{\prime} \mathscr{L}\left[u^{\prime} ; \partial^{\prime} u^{\prime}\right]\left(x^{\prime}\right)-\int_{\Omega} d^{4} x \mathscr{L}[u ; \partial u](x) \\
& =\int_{\Omega} d^{4} x\left(\delta \mathscr{L}+\mathscr{L} \partial_{\mu} \delta x^{\mu}\right) .
\end{aligned}
$$

Essa expressão pode ser escrita de forma conveniente definindo a derivada de Euler da densidade lagrangiana,

$$
\frac{\delta \mathscr{L}}{\delta u^{A}}:=\frac{\partial \mathscr{L}}{\partial u^{A}}-\partial_{\mu} \frac{\partial \mathscr{L}}{\partial\left(\partial_{\mu} u^{A}\right)},
$$

e a quantidade:

$$
\Theta^{\mu}{ }_{\nu}:=\frac{\partial \mathscr{L}}{\partial\left(\partial_{\mu} u^{A}\right)} \partial_{\nu} u^{A}-\delta^{\mu}{ }_{\nu} \mathscr{L} .
$$

Então a equação 150 é equivalente a:

$$
\begin{aligned}
\delta \mathscr{A}[u]= & \int_{\Omega} d^{4} x\left\{\frac{\delta \mathscr{L}}{\delta u^{A}}\left(\delta u^{A}-\partial_{\mu} u^{A} \delta x^{\mu}\right)\right. \\
& \left.-\partial_{\mu}\left[\Theta^{\mu}{ }_{\nu} \delta x^{\nu}-\frac{\partial \mathscr{L}}{\partial\left(\partial_{\mu} u^{A}\right)} \delta u^{A}\right]\right\} .
\end{aligned}
$$

Escrita nesta forma é possível a aplicação direta de princípios variacionais à integral de ação:

Princípio da ação estacionária (de OstrogradskiiHamilton): Campo físico é aquele cuja forma funcional $\left(\delta x^{\mu}=0\right)$ é tal que, entre duas configurações fixas do sistema $\left(\left.\delta u^{A}(x)\right|_{\partial \Omega}=0\right)$, a integral de ação adota um valor estacionário $(\delta \mathscr{A}[u]=0)$ qualquer que seja a região $\Omega$.

As equações de Euler-Lagrange são consequência do princípio da ação estacionária, pois a equação 153 implica que:

$$
\frac{\delta \mathscr{L}}{\delta u^{A}}=0 ; \quad A=1, \cdots, N
$$

Primeiro teorema de Nöther: Se a integral de ação de um dado sistema físico é invariante, até termos de superfície, em relação a certo grupo de Lie $G_{r}$ de $r$ parâmetros, então $r$ combinações linearmente independentes de derivadas de Euler da densidade lagrangiana são iguais a divergências.

Prova: Denotemos por $\epsilon^{a}(a=1, \cdots, r)$ os parâmetros do grupo $G_{r}$, e consideremos que a transformação age sobre as coordenadas e campos mediante as representações $X_{a}$ e $I_{a}$, respectivamente; ou seja:

$$
\delta x^{\mu}=\epsilon^{a}\left[X_{a} x\right]^{\mu}, \quad \delta u^{A}=\epsilon^{a}\left[I_{a} u\right]^{A} .
$$

Por hipótese do teorema, a ação é invariante, até termos de superfície, frente às transformações da equação 155 : $\int_{\partial \Omega} d^{4} x \partial_{\mu}\left(\epsilon^{a} \delta_{a} \Omega^{\mu}\right)$. Portanto, na equação 153:

$$
\begin{gathered}
\int_{\Omega} d^{4} x \\
\left\{\frac{\delta \mathscr{L}}{\delta u^{A}}\left(\left[I_{a} u\right]^{A}-\partial_{\mu} u^{A}\left[X_{a} x\right]^{\mu}\right)\right. \\
\left.-\partial_{\mu} J_{a}^{\mu}\right\} \epsilon^{a}=0,
\end{gathered}
$$

com:

$$
J_{a}^{\mu}:=\Theta_{\nu}^{\mu}\left[X_{a} x\right]^{\nu}-\frac{\partial \mathscr{L}}{\partial\left(\partial_{\mu} u^{A}\right)}\left[I_{a} u\right]^{A}-\delta_{a} \Omega^{\mu} .
$$

Ora a independência linear dos parâmetros do grupo $G_{r}$, $\epsilon^{a}$, nos permite escrever a tese do teorema:

$$
\frac{\delta \mathscr{L}}{\delta u^{A}}\left(\left[I_{a} u\right]^{A}-\partial_{\mu} u^{A}\left[X_{a} x\right]^{\mu}\right)=\partial_{\mu} J_{a}^{\mu} ; a=1, \cdots, r
$$

quod erat demonstrandum.

Para campos físicos, as identidades da equação 158 se tornam $r$ leis de conservação das densidades de corrente $J^{\mu}{ }_{a}$, pois o lado esquerdo delas se anula por ser proporcional às equações de Euler-Lagrange. Como elas regem apenas sob a imposição dos campos serem físicos, a elas se chama «leis de conservação débeis». Neste caso, as correspondentes cargas conservadas, chamadas «cargas de Nöther», são obtidas por integração da densidade de corrente sobre uma superfície $\Sigma$ com elemento de área $d^{3} \sigma_{\mu}=d^{3} \sigma n_{\mu}$, com $n_{\mu}$ o vetor normal a $\Sigma$ :

$$
Q_{a}:=\int_{\Sigma} d^{3} \sigma(x) n_{\mu} J_{a}^{\mu}(x) .
$$

Na dinâmica da frente de luz, a superfície $\Sigma$ é o plano nulo $x^{(+)}=x_{0}^{(+)}=$constante. Este plano é, como já foi observado, gerado pelos vetores $e_{(\perp)}$ e $e_{(-)}$, $\mathrm{e}$, portanto, tem vetor normal $\mathrm{n}=e_{(-)}$, ortogonal a eles [note que o vetor $e_{(+)}$, que poderíamos pensar inicialmente que fosse o vetor normal, falha, pois não é ortogonal a $e_{(-)}$, segundo a equação 142$]$ ]. Substituindo 
na equação 159 :

$$
\begin{gathered}
Q_{a}=\int_{x^{(+)}=x_{0}^{(+)}} d^{3} \underline{\boldsymbol{x}} e_{(-) \mu} J_{a}^{\mu}(x) \\
=\int_{x^{(+)}=x_{0}^{(+)}} d^{3} \underline{\boldsymbol{x}} J^{(+)}{ }_{a}(x) ; \\
\frac{d Q_{a}}{d x^{(+)}}=0 .
\end{gathered}
$$

Tipicamente, as densidades de corrente são formas bilineares dos campos, então as cargas de Nöther existirão (a integral que as define será convergente) se os campos, assim como suas derivadas transversais e longitudinal, são de quadrado integrável no plano nulo. Essas condições assintóticas requeridas para a existência das cargas de Nöther, vemos, incluem a condição assintótica da equação (133) que substituia os dados iniciais na superfície característica $x^{(-)}=$constante.

Consideremos, particularmente, o gerador das translações temporais no plano nulo, isto é, o hamiltoniano. Consideremos a seguinte translação em forma covariante: $x^{\prime \mu}=x^{\mu}+\epsilon^{\mu}, u^{\prime A}\left(x^{\prime}\right)=u^{A}(x)$, ou:

$$
\left[X_{\nu} x\right]^{\mu}=\delta_{\nu}^{\mu}, \quad\left[I_{\nu} u\right]^{A}=0 .
$$

Também, como a densidade lagrangiana é escalar frente a transformações do grupo de Poincaré, teremos que $\delta \mathscr{L}=0$. Isto na equação 150 implica que a integral de ação é invariante sob translações, pois os parâmetros $\epsilon^{\mu}$ são constantes, e assim $\delta_{\nu} \Omega^{\mu}=0$. Substituindo a equação 162 na equação 157, obtemos a densidade de corrente conservada:

$$
J_{\nu}^{\mu}=\Theta^{\mu}{ }_{\sigma}\left[X_{\nu} x\right]^{\sigma}=\Theta^{\mu}{ }_{\nu},
$$

que é chamada «tensor de energia-momento». A carga de Nöther que lhe corresponde, com respeito ao plano nulo, é o «vetor de energia-momento» com componentes [vide a equação 160]:

$$
P_{\nu}=\int_{x^{(+)}=x_{0}^{(+)}} d^{3} \underline{\boldsymbol{x}} \Theta^{(+)}{ }_{\nu}
$$

As componentes invariantes desse vetor são obtidas projetando seu índice $\nu$ com o campo de tétradas da dinâmica da frente de luz:

$$
P_{(a)}=e_{(a)}{ }^{\nu} P_{\nu}=\int d^{3} \underline{\boldsymbol{x}} e_{(a)}{ }^{\nu} \Theta_{\nu}^{(+)}=\int d^{3} \underline{\boldsymbol{x}} \Theta^{(+)}{ }_{(a)} .
$$

Assim, o momento invariante no plano nulo é:

$$
P_{(\perp)}=\int d^{3} \underline{\boldsymbol{x}}^{(+)}{ }_{(\perp)}, \quad P_{(-)}=\int d^{3} \underline{\boldsymbol{x}}^{(+)}{ }_{(-)},
$$

enquanto que o hamiltoniano invariante tem expressão:

$$
\begin{aligned}
H_{P N} \equiv P_{(+)} & =\int d^{3} \underline{\boldsymbol{x}} \Theta_{(+)}^{(+)} \\
& =\int d^{3} \underline{\boldsymbol{x}} \Theta_{(-)(+)} .
\end{aligned}
$$

E sobre a teoria lagrangiana geral do campo clássico basta o que foi dito. O segundo teorema de Nöther, referente à localização das simetrias, isto é, à ampliação do grupo de simetrias $G_{r}$ que se obtém ao fazer os parâmetros do grupo depender do ponto $x$ do espaçotempo, leva imediatamente às teorias de calibre e, assim, aos campos em interação. Isto o deixamos para um próximo trabalho. Focaremos nossa atenção agora em estudar os diversos campos particulares usados na modelagem da matéria. E uma vez clara a ideia subjacente às coordenadas invariantes, deixaremos de escrever os índices entre parênteses.

\section{Campo escalar}

Seja $\left(\varphi, \varphi^{*}\right)$ um campo escalar complexo, cujas equações de movimento são as de Klein-Gordon-Fock:

$$
\left(\square+m^{2}\right) \varphi(x)=0 ; \quad\left(\square+m^{2}\right) \varphi^{*}(x)=0 .
$$

Como não há outras equações além dessas, o problema de Goursat para o campo escalar é simplesmente aquele que já estudamos na seção 3. A densidade lagrangiana que deriva nessas equações é a seguinte:

$$
\mathscr{L}_{\varphi}=\partial_{\mu} \varphi^{*} \partial^{\mu} \varphi-m^{2} \varphi^{*} \varphi
$$

A substituição dela na equação (152) nos permite obter o tensor de energia-momento desse campo:

$$
\begin{aligned}
\Theta^{\mu}{ }_{\nu}= & \frac{\partial \mathscr{L}_{\varphi}}{\partial\left(\partial_{\mu} \varphi\right)} \partial_{\nu} \varphi+\frac{\partial \mathscr{L}_{\varphi}}{\partial\left(\partial_{\mu} \varphi^{*}\right)} \partial_{\nu} \varphi^{*}-\delta^{\mu}{ }_{\nu} \mathscr{L}_{\varphi} \\
= & \partial^{\mu} \varphi^{*} \partial_{\nu} \varphi+\partial_{\nu} \varphi^{*} \partial^{\mu} \varphi-\delta^{\mu}{ }_{\nu} \partial_{\sigma} \varphi^{*} \partial^{\sigma} \varphi \\
& +m^{2} \delta^{\mu}{ }_{\nu} \varphi^{*} \varphi .
\end{aligned}
$$

O hamiltoniano invariante do plano nulo será portanto [equação 166] ]:

$$
H_{P N ; \varphi}=\int d^{3} \underline{\boldsymbol{x}}\left[\partial_{\perp} \varphi^{*} \partial_{\perp} \varphi+m^{2} \varphi^{*} \varphi\right] .
$$

Consideremos agora a equação de Klein-Gordon-Fock [equação (168)] à que o campo escalar obedece. Nas coordenadas do plano nulo, o operador D'Alembertiano adota a forma:

$$
\square=2 \partial_{+} \partial_{-}-\left(\partial_{\perp}\right)^{2} ;\left(\partial_{\perp}\right)^{2}=\partial_{1}^{2}+\partial_{2}^{2} .
$$

A solução dela será facilmente encontrada ao tomarmos a transformada de Fourier do campo:

$$
\begin{aligned}
\varphi(x)= & (2 \pi)^{-3 / 2} \int d^{4} p \hat{\varphi}(p) e^{-i p x} \\
= & (2 \pi)^{-3 / 2} \int d p_{+} d p_{-} d^{2} p_{\perp} \hat{\varphi}(p) \\
& \times e^{-i\left(p_{+} x^{+}+p_{-} x^{-}+p_{\perp} x^{\perp}\right)},
\end{aligned}
$$


e daí que:

$$
\begin{gathered}
\left(\square+m^{2}\right) \varphi(x)=-(2 \pi)^{-3 / 2} \int d p_{+} d p_{-} d^{2} p_{\perp} \\
\times\left(2 p_{+} p_{-}-\omega_{p}^{2}\right) \hat{\varphi}(p) e^{-i p x}=0 ;
\end{gathered}
$$

aqui: $\omega_{p}^{2}:=m^{2}+\left(p_{\perp}\right)^{2}$. Vemos que deverá ser satisfeita a relação: $\left(2 p_{+} p_{-}-\omega_{p}^{2}\right) \hat{\varphi}(p)=0$, cuja solução distribucional é dada por 62:

$$
\begin{aligned}
\hat{\varphi}(p) & =\delta\left(2 p_{+} p_{-}-\omega_{p}^{2}\right) \varphi(p) \\
& =\frac{1}{\left|2 p_{-}\right|} \delta\left(p_{+}-\frac{\omega_{p}^{2}}{2 p_{-}}\right) \varphi(p),
\end{aligned}
$$

com $\varphi(p)$ uma função arbitrária. Substituindo na equação (173) e separando a região de integração da variável $p_{+}$no domínio positivo e no negativo:

$$
\begin{aligned}
\varphi(x)= & (2 \pi)^{-3 / 2} \int d^{2} p_{\perp} \int_{-\infty}^{+\infty} d p_{-} \\
& \times\left\{\int_{-\infty}^{0} \frac{d p_{+}}{\left|2 p_{-}\right|} \delta\left(p_{+}-\frac{\omega_{p}^{2}}{2 p_{-}}\right) \varphi(p) e^{-i p x}\right. \\
& \left.+\int_{0}^{+\infty} \frac{d p_{+}}{\left|2 p_{-}\right|} \delta\left(p_{+}-\frac{\omega_{p}^{2}}{2 p_{-}}\right) \varphi(p) e^{-i p x}\right\} .
\end{aligned}
$$

Façamos na primeira integral a substituição $p \rightarrow-p$; usando então a propriedade de simetria da distribuição delta de Dirac, isto é, que $\delta(x)=\delta(-x)$, obtemos que:

$$
\begin{aligned}
\varphi(x)= & (2 \pi)^{-3 / 2} \int d^{2} p_{\perp} \int_{-\infty}^{+\infty} d p_{-} \int_{0}^{+\infty} \frac{d p_{+}}{\left|2 p_{-}\right|} \\
& \times \delta\left(p_{+}-\frac{\omega_{p}^{2}}{2 p_{-}}\right)\left[\varphi(-p) e^{i p x}+\varphi(p) e^{-i p x}\right] .
\end{aligned}
$$

Como é indicado pelos limites de integração, aqui é $p_{+}>0$. Mas o suporte da distribuição delta de Dirac exige que seja $p_{+}=\omega_{p}^{2} / 2 p_{-}$, e como $\omega_{p}^{2}>0$, é forçoso que seja $p_{-}>0$. Assim é que devemos introduzir a função de Heaviside $\Theta\left(p_{-}\right)$e então escrever:

$$
\begin{aligned}
\varphi(x)= & (2 \pi)^{-3 / 2} \int d^{2} p_{\perp} \int d p_{-} \int_{0}^{+\infty} \frac{d p_{+}}{\left|2 p_{-}\right|} \Theta\left(p_{-}\right) \\
& \times \delta\left(p_{+}-\frac{\omega_{p}^{2}}{\left|2 p_{-}\right|}\right)\left[\varphi(-p) e^{i p x}+\varphi(p) e^{-i p x}\right] .
\end{aligned}
$$

Integrando finalmente a variável $p_{+}$:

$$
\begin{aligned}
\varphi(x)= & (2 \pi)^{-3 / 2} \int \frac{d^{3} \underline{p}}{\left|2 p_{-}\right|} \Theta\left(p_{-}\right) \\
& \times\left.\left[\varphi(-p) e^{i p x}+\varphi(p) e^{-i p x}\right]\right|_{p_{+}=E},
\end{aligned}
$$

em que temos escrito: $d^{3} \boldsymbol{p}:=d^{2} p_{\perp} d p_{-}$, e com a energia $E$ definida segundo: $E:=\omega_{p}^{2} /\left|2 p_{-}\right|>0$. Note que essa expressão é relativisticamente correta, pois a medida de integração é invariante sob transformações de Poincaré:

$$
\begin{gathered}
\int \frac{d^{2} p_{\perp} d p_{-}}{\left|2 p_{-}\right|} \Theta\left(p_{-}\right)=\int \frac{d^{4} p}{\left|2 p_{-}\right|} \Theta\left(p_{-}\right) \delta\left(p_{+}-E\right) \\
=\int d^{4} p \Theta\left(p_{-}\right) \delta\left(2 p_{+} p_{-}-\omega_{p}^{2}\right) \\
=\int d^{4} p \Theta\left(p_{-}\right) \delta\left(p^{2}-m^{2}\right) .
\end{gathered}
$$

Lembremos, por outro lado, que $\Theta\left(p_{-}\right)$é invariante sob transformações de Poincaré ortócronas, pois $p_{-}$é uma coordenada do tipo-luz.

É habitual realizar a normalização seguinte ${ }^{11}[53$ :

$$
\varphi(\underline{\boldsymbol{p}}):=\frac{\varphi(p)}{\sqrt{\left|2 p_{-}\right|}}, \quad \varphi(-\underline{\boldsymbol{p}}):=\frac{\varphi(-p)}{\sqrt{\left|2 p_{-}\right|}},
$$

com a qual:

$$
\begin{aligned}
\varphi(x)= & (2 \pi)^{-3 / 2} \int \frac{d^{3} \underline{\underline{p}}}{\sqrt{\left|2 p_{-}\right|}} \Theta\left(p_{-}\right) \\
& \times\left.\left[\varphi(\underline{\boldsymbol{p}}) e^{-i p x}+\varphi(-\underline{\boldsymbol{p}}) e^{i p x}\right]\right|_{p_{+}=E} .
\end{aligned}
$$

Finalmente, se o campo clássico é real, isto é, se $\varphi(x)=\varphi^{*}(x)$, então sobre as amplitudes dos diferentes modos deve-se impor a condição:

$$
\varphi(-p)^{*}=\varphi(p), \quad \varphi(p)^{*}=\varphi(-p) .
$$

Assim, a expansão em série de Fourier do campo escalar real é:

$$
\begin{aligned}
\varphi(x)= & (2 \pi)^{-3 / 2} \int \frac{d^{3} \underline{\boldsymbol{p}}}{\sqrt{\left|2 p_{-}\right|}} \Theta\left(p_{-}\right) \\
& \times\left.\left[\varphi(\underline{\boldsymbol{p}}) e^{-i p x}+\varphi(\underline{\boldsymbol{p}})^{*} e^{i p x}\right]\right|_{p_{+}=E} .
\end{aligned}
$$

\section{Campo de Dirac}

Consideremos agora o campo fermiônico de Dirac $(\psi, \bar{\psi})$. As equações de Dirac sã ${ }^{12}$.

$$
(i \not \partial-m) \psi(x)=0, \bar{\psi}(x)(i \overleftarrow{\not}+m)=0
$$

\footnotetext{
11 O motivo dela é a simplificação dos geradores do grupo de Poincaré quando escritos em função das amplitudes $\varphi(p)$; tal normalização se torna importante na teoria quântica, pois determina as relações de comutação dos operadores de emissão e absorção; isto, no entanto, não será discutido no artigo presente. 12 A definição do adjunto de Dirac $\bar{\psi}$ é obtida da forma que segue: A equação de Dirac nas coordenadas do plano nulo é:
}

$$
\left[i\left(\gamma^{+} \partial_{+}+\gamma^{-} \partial_{-}+\gamma^{\perp} \partial_{\perp}\right)-m\right] \psi(x)=0 .
$$


as quais provêm da densidade lagrangiana:

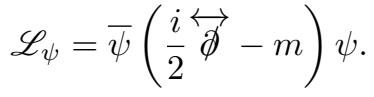

O tensor de energia-momento desse campo é obtido por substituição dessa densidade lagrangiana na equação 152):

$$
\begin{aligned}
\Theta^{\mu}{ }_{\nu} & =\frac{\partial \mathscr{L}_{\psi}}{\partial\left(\partial_{\mu} \psi\right)} \partial_{\nu} \psi+\partial_{\nu} \bar{\psi} \frac{\partial \mathscr{L}_{\varphi}}{\partial\left(\partial_{\mu} \bar{\psi}\right)}-\delta^{\mu}{ }_{\nu} \mathscr{L}_{\psi} \\
= & \frac{i}{2}\left(\bar{\psi} \gamma^{\mu} \partial_{\nu} \psi-\partial_{\nu} \bar{\psi} \gamma^{\mu} \psi\right)-\delta^{\mu}{ }_{\nu} \bar{\psi}\left(\frac{i}{2} \overleftrightarrow{\not \partial}-m\right) \psi
\end{aligned}
$$

O hamiltoniano invariante do plano nulo será portanto:

$$
\begin{aligned}
& H_{P N ; \psi} \\
& =\int d^{3} \underline{\boldsymbol{x}}\left[-\frac{i}{2} \bar{\psi}\left(\gamma^{-\overleftrightarrow{\partial}_{-}}+\gamma^{\perp} \overleftrightarrow{\partial}_{\perp}\right) \psi+m \bar{\psi} \psi\right]
\end{aligned}
$$

\subsection{Componentes dinâmicas e não-dinâmicas}

Investiguemos em detalhe a equação de Dirac [equação (185]). Nas coordenadas do plano nulo, separando a derivada temporal $\partial_{+}$:

$$
i \gamma^{+} \partial_{+} \psi=\left(m-i \gamma^{-} \partial_{-}-i \gamma^{\perp} \partial_{\perp}\right) \psi .
$$

A matriz $\gamma^{+}$não pode ser invertida ${ }^{13}$ pois ela tem determinante nulo (isto pode ser visto, bem por cálculo direto, por exemplo, na representação quiral ou em qualquer outra, bem pelo fato de que seu quadrado é nulo devido à relação de anti-comutação a que as matrizes de Dirac satisfazem -o que indica que também a matriz $\gamma^{-}$ é não invertível-). Portanto, poderemos isolar a derivada temporal de forma a escrever a equação 189 na forma da equação de Schrödinger somente pela definição dos projetores:

$$
\Lambda_{ \pm}:=\frac{1}{2}\left(1 \pm \gamma^{0} \gamma^{3}\right)=\frac{1}{\sqrt{2}} \gamma^{0} \gamma^{ \pm}
$$

Tomando o adjunto no espaço das matrizes (isto é, o complexo conjugado seguido pela transposição), as derivadas, sendo reais, não mudam: $\partial_{a}^{\dagger}=\partial_{a}(a=+, \perp,-)$. Por outro lado: $\left(\gamma^{a}\right)^{\dagger}=$ $\gamma^{0} \gamma^{a} \gamma^{0}(a=+, \perp,-)$, de forma que a equação muda para (fatorando o sinal negativo, então removendo-o):

$$
\psi(x)^{\dagger}\left[i\left(\gamma^{0} \gamma^{+} \gamma^{0} \partial_{+}+\gamma^{0} \gamma^{-} \gamma^{0} \partial_{-}+\gamma^{0} \gamma^{\perp} \gamma^{0} \partial_{\perp}\right)+m\right]=0 .
$$

Multiplicando pela direita por $\gamma^{0}$ e usando que $\left(\gamma^{0}\right)^{2}=1$ :

$$
\psi(x)^{\dagger} \gamma^{0}\left[i\left(\gamma^{+} \partial_{+}+\gamma^{-} \partial_{-}+\gamma^{\perp} \partial_{\perp}\right)+m\right]=0,
$$

do qual concluímos que o espinor adjunto de Dirac na dinâmica da frente de luz continúa a ser:

$$
\bar{\psi}(x):=\psi(x)^{\dagger} \gamma^{0} .
$$

13 A expressão das matrizes de Dirac no plano nulo e na representação de Weyl é mostrada no apêndice B os quais exibem as seguintes propriedades:

$$
\Lambda_{ \pm}^{2}=\Lambda_{ \pm} ; \Lambda_{ \pm} \Lambda_{\mp}=0 ; \Lambda_{+}+\Lambda_{-}=1,
$$

como é possível provar por cálculo direto. Da mesma forma podem ser mostradas as igualdades que seguem:

$$
\begin{gathered}
\Lambda_{ \pm} \gamma^{0}=\gamma^{0} \Lambda_{\mp}, \quad \Lambda_{+} \gamma^{0} \gamma^{-}=0 \\
\Lambda_{-} \gamma^{0} \gamma^{-}=\gamma^{0} \gamma^{-} \Lambda_{-}, \quad \Lambda_{ \pm} \gamma^{0} \gamma^{\perp}=\gamma^{0} \gamma^{\perp} \Lambda_{\mp} .
\end{gathered}
$$

Finalmente, definimos as duas projeções do campo de Dirac segundo:

$$
\psi_{ \pm}:=\Lambda_{ \pm} \psi ; \quad \psi=\psi_{+}+\psi_{-} .
$$

Com isto, multiplicando a equação 189 por $\gamma^{0}$ pela esquerda e usando a definição de $\Lambda_{+}$, obtemos:

$$
i \sqrt{2} \partial_{+} \psi_{+}=\gamma^{0}\left(m-i \gamma^{-} \partial_{-}-i \partial^{\perp} \partial_{\perp}\right) \psi .
$$

Multiplicando pela esquerda por $\Lambda_{+}$e usando as propriedades mostradas na equação 192 , chegamos a:

$$
i \sqrt{2} \partial_{+} \psi_{+}=\gamma^{0}\left(m-i \gamma^{\perp} \partial_{\perp}\right) \psi_{-} .
$$

Análogamente, multiplicando a equação 194 por $\Lambda_{-}$ pela esquerda e usando a equação 192 :

$$
i \sqrt{2} \partial_{-} \psi_{-}=\gamma^{0}\left(m-i \gamma^{\perp} \partial_{\perp}\right) \psi_{+} .
$$

A equação 195 é uma equação dinâmica para a componente $\psi_{+}$; ela é portanto chamada «componente dinâmica» do campo de Dirac. A equação (196), por outro lado, não é uma equação dinâmica para $\psi_{-}$, visto que nenhuma derivada temporal aparece nela; $\psi_{-}$é chamada «componente não-dinâmica», e a equação (196) é uma equação de vínculo, a qual pode ser invertida da forma seguinte, explicitando o fato de que $\psi_{-}$é automaticamente conhecida uma vez que o é $\psi_{+}$:

$$
\psi_{-}=\frac{1}{i \sqrt{2} \partial_{-}} \gamma^{0}\left(m-i \gamma^{\perp} \partial_{\perp}\right) \psi_{+} .
$$

Este vínculo pode ser usado para eliminar $\psi_{-}$na equação 195. Encontramos que a equação de movimento da componente dinâmica do campo de Dirac não é senão a equação de Klein-Gordon-Fock:

$$
\left(\square+m^{2}\right) \psi_{+}=0 .
$$

\subsection{Problema de Goursat}

O problema de Goursat para o campo de Dirac pode ser abordado da mesma forma que na seção 3 Solucionando primeiro o problema de Cauchy (na dinâmica instantânea) e passando depois à dinâmica do plano nulo por aplicação do teorema de Gauss num volume convenientemente escolhido. Como, no entanto, este caminho já foi percorrido anteriormente -e não encontraremos dificuldades em percorrê-lo para o caso presente se assim o 
desejarmos-, é mais instrutivo oferecer uma outra forma de obter a desejada solução. Tal o faremos explorando a divisão recém feita entre as componentes dinâmicas e as não-dinâmicas. Devido a que as componentes dinâmicas satisfazem a equação de Klein-Gordon-Fock [equação (198)], seu problema de Goursat tem a solução dada na equação 134, que por uma integração por partes pode ser escrita da seguinte forma:

$$
\psi_{+}(x)=\int_{y^{+}=y_{0}^{+}} d^{3} \underline{\boldsymbol{y}} 2 \partial_{-}^{x} D(x-y) \psi_{+}(y) .
$$

As componentes não-dinâmicas são então determinadas pela substituição da equação 199 na (197), da qual obtemos:

$$
\begin{aligned}
\psi_{-}(x) & =-i \sqrt{2} \\
\times & \int_{y^{+}=y_{0}^{+}} d^{3} \underline{\boldsymbol{y}} \gamma^{0}\left(m-i \gamma^{\perp} \partial_{\perp}^{x}\right) D(x-y) \psi_{+}(y) .
\end{aligned}
$$

E pelo uso das definições mostradas na equação (193):

$$
\begin{gathered}
\psi(x)=\int_{y^{+}=y_{0}^{+}} d^{3} \underline{\boldsymbol{y}}\left[2 \partial_{-}^{x}\right. \\
\left.-i \sqrt{2} \gamma^{0}\left(m-i \gamma^{\perp} \partial_{\perp}^{x}\right)\right] D(x-y) \Lambda_{+} \psi(y) .
\end{gathered}
$$

Usando então as equações 190-192, assim como o fato, já mencionado, de ser $\left(\gamma^{+}\right)^{2}=0$, vemos que é possível escrever a equação 201 na forma sucinta:

$$
\psi(x)=-i \int_{y^{+}=y_{0}^{+}} d^{3} \underline{\boldsymbol{y}} S(x-y) \gamma^{+} \psi(y),
$$

com a distribuição $S(x)$ definida como:

$$
S(x):=(i \not \partial+m) D(x) .
$$

Tendo encontrado a solução -o que, já o dissemos, imediatamente implica que ela é única- podemos afirmar que o problema de Goursat para o campo de Dirac está bem definido. Por outro lado, no problema de Cauchy seria necessário conhecer o campo $\psi(0 ; \boldsymbol{x})$, que faz um total de quatro funções, pois as derivadas dele não serão necessárias devido a que a equação de Dirac é uma equação de primeira ordem; o mesmo número de dados é necessário na solução que apresentamos e na qual usamos a equação 199, que implica o conhecimento de $\psi_{+}$em $x^{+}=0$ e $x^{-}=x_{0}^{-}$, fazendo igualmente um total de quatro funções -sendo que essa última tem sido substituída por uma condição assintótica, o que não muda o número de dados iniciais--

\subsection{Espinores de polarização}

Como cada uma das componentes do campo de Dirac, $\psi_{a}(x)$, satisfaz a equação de Klein-Gordon-Fock,

$$
\left(\square+m^{2}\right) \psi_{a}(x)=0,
$$

cada uma delas irá ter uma expressão semelhante àquela da equação 182):

$$
\begin{aligned}
\psi_{a}(x)= & (2 \pi)^{-3 / 2} \int \frac{d^{3} \underline{\boldsymbol{p}}}{\sqrt{\left|2 p_{-}\right|}} \Theta\left(p_{-}\right) \\
& \times\left.\left[\psi_{a}(\underline{\boldsymbol{p}}) e^{-i p x}+\psi_{a}(-\underline{\boldsymbol{p}}) e^{i p x}\right]\right|_{p_{+}=E}
\end{aligned}
$$

As amplitudes espinoriais $\psi(\underline{\boldsymbol{p}})$ que aqui aparecem devem ser tais que $\psi_{a}(x)$ satisfaça a equação de Dirac [equação (185]): Elas carregam a informação «adicional» não contida na equação de Klein-Gordon-Fock, isto é, a informação dos vínculos da teoria. Logo tais amplitudes definem os possíveis estados de polarização do campo de Dirac. Substituindo a equação 205 na 185 :

$$
\begin{aligned}
0 & =(i \not \partial-m) \psi(x)=(2 \pi)^{-3 / 2} \int \frac{d^{3} \underline{\boldsymbol{p}}}{\sqrt{\left|2 p_{-}\right|}} \Theta\left(p_{-}\right) \\
& \times\left.\left[(\not p-m) \psi(\underline{\boldsymbol{p}}) e^{-i p x}-(\not p+m) \psi(-\underline{\boldsymbol{p}}) e^{i p x}\right]\right|_{p_{+}=E} .
\end{aligned}
$$

Daqui concluímos que, uma vez que as soluções de freqüências positivas e as de freqüências negativas são linearmente independentes, deverão ser verificadas as equações:

$$
(\not p-m) \psi(\underline{\boldsymbol{p}})=0 ; \quad(\not p+m) \psi(-\underline{\boldsymbol{p}})=0 .
$$

Apresentaremos a seguir a solução para essas equações, inspirados na Ref. [23].

Comecemos com:

$$
(\not p-m) u(\underline{\boldsymbol{p}})=0 .
$$

De forma semelhante a que foram obtidas as equações 195 e 196), encontramos que:

$$
\begin{aligned}
& \sqrt{2} p_{+} u_{+}=\gamma^{0}\left(m-\gamma^{\perp} p_{\perp}\right) u_{-}, \\
& \sqrt{2} p_{-} u_{-}=\gamma^{0}\left(m-\gamma^{\perp} p_{\perp}\right) u_{+},
\end{aligned}
$$

lembrando que é $p_{+}=E$ [vide a equação (206)]. A estratégia para solucionar essas equações será escrever uma base para uma das projeções do espinor e usar, então, a 209 ou a 210 para encontrar a outra. No apêndice $B$ mostra-se a forma explícita dos projetores [equação [300] ], da qual observamos que uma base para u_ será:

$$
u_{-}^{(1)}=\left(\begin{array}{l}
0 \\
1 \\
0 \\
0
\end{array}\right), \quad u_{-}^{(-1)}=\left(\begin{array}{l}
0 \\
0 \\
1 \\
0
\end{array}\right) \text {. }
$$

Os espinores $u_{+}$correspondentes são obtidos usando a equação 209):

$$
u_{+}^{(1)}=\frac{1}{\sqrt{2} E}\left(\begin{array}{c}
-p_{1}+i p_{2} \\
0 \\
0 \\
m
\end{array}\right),
$$




$$
u_{+}^{(-1)}=\frac{1}{\sqrt{2} E}\left(\begin{array}{c}
m \\
0 \\
0 \\
p_{1}+i p_{2}
\end{array}\right) \text {. }
$$

Ora, a equação 193 implica que a solução completa é obtida somando as componentes $u_{-}$e $u_{+}$, o que leva a obter:

$$
\begin{aligned}
& u_{1}=\frac{a_{0}}{\sqrt{2} E}\left(\begin{array}{c}
-p_{1}+i p_{2} \\
\sqrt{2} E \\
0 \\
m
\end{array}\right), \\
& u_{-1}=\frac{a_{0}}{\sqrt{2} E}\left(\begin{array}{c}
m \\
0 \\
\sqrt{2} E \\
p_{1}+i p_{2}
\end{array}\right) .
\end{aligned}
$$

Nessas expressões, $a_{0}$ é um fator de normalização, que escolheremos convenientemente como sendd 14

$$
a_{0}=\sqrt{\frac{E}{\sqrt{2}\left|p_{-}\right|}} .
$$

Com isto:

$$
\begin{gathered}
u_{1}=\frac{1}{\sqrt{2 E} \sqrt{\sqrt{2}\left|p_{-}\right|}}\left(\begin{array}{c}
-p_{1}+i p_{2} \\
\sqrt{2} E \\
0 \\
m
\end{array}\right), \\
u_{-1}=\frac{1}{\sqrt{2 E} \sqrt{\sqrt{2}\left|p_{-}\right|}}\left(\begin{array}{c}
m \\
0 \\
\sqrt{2} E \\
p_{1}+i p_{2}
\end{array}\right) .
\end{gathered}
$$

Assim é que podemos diretamente calcular:

$$
\begin{aligned}
& u_{1} u_{1}^{\dagger}+u_{-1} u_{-1}^{\dagger}=\frac{1}{2 E \sqrt{2}\left|p_{-}\right|} \\
& \times\left(\begin{array}{cc}
p_{\perp}^{2}+m^{2} & -\sqrt{2} E\left(p_{1}-i p_{2}\right) \\
-\sqrt{2} E\left(p_{1}+i p_{2}\right) & 2 E^{2} \\
\sqrt{2} m E & 0 \\
0 & \sqrt{2} m E \\
\sqrt{2} m E & 0 \\
0 & \sqrt{2} m E \\
2 E^{2} & \sqrt{2} E\left(p_{1}-i p_{2}\right) \\
\sqrt{2} E\left(p_{1}+i p_{2}\right) & p_{\perp}^{2}+m^{2}
\end{array}\right) .
\end{aligned}
$$

\footnotetext{
$\overline{14} \mathrm{O}$ mesmo que na normalização das amplitudes do campo escalar na equação 181, essa escolha é livre -faça o leitor a escolha que lhe aprouver-. Se refletirá, por exemplo, nas regras de soma que estamos prestes a obter [vide as equações 220 e 227]].
}

Multiplicando pela direita por $\gamma^{0}$ :

$$
\begin{aligned}
& u_{1} \bar{u}_{1}+u_{-1} \bar{u}_{-1}=\frac{1}{\left|2 p_{-}\right|} \\
& \times\left(\begin{array}{cccc}
m & 0 & \sqrt{2}\left|p_{-}\right| & -\left(p_{1}-i p_{2}\right) \\
0 & m & -\left(p_{1}+i p_{2}\right) & \sqrt{2} E \\
\sqrt{2} E & p_{1}-i p_{2} & m & 0 \\
p_{1}+i p_{2} & \sqrt{2}\left|p_{-}\right| & 0 & m
\end{array}\right) .
\end{aligned}
$$

E portanto, lembrando da representação de Weyl das matrizes de Dirac [apêndice B], reconhecemos que a equação anterior se iguala a:

$$
\sum_{s= \pm 1} u_{s} \bar{u}_{s}=\frac{E \gamma^{+}+\left|p_{-}\right| \gamma^{-}+p_{\perp} \gamma^{\perp}+m}{\left|2 p_{-}\right|}
$$

Demos agora, por método análogo, solução à segunda das equações 207):

$$
(\not p+m) v=0,
$$

da qual se segue, após projeção com $\Lambda_{ \pm}$, que as componentes do espinor $v$ se relacionam por:

$$
v_{+}=-\frac{\gamma^{0}\left(m+p_{\perp} \gamma^{\perp}\right)}{\sqrt{2} E} v_{-} .
$$

Usando a mesma base apresentada na equação 211 para os espinores $v_{-}$e usando a relação da equação 222, obtemos:

$$
\begin{gathered}
v_{+}^{(1)}=-\frac{1}{\sqrt{2} E}\left(\begin{array}{c}
p_{1}-i p_{2} \\
0 \\
0 \\
m
\end{array}\right), \\
v_{+}^{(-1)}=-\frac{1}{\sqrt{2} E}\left(\begin{array}{c}
m \\
0 \\
0 \\
-p_{1}-i p_{2}
\end{array}\right) .
\end{gathered}
$$

A equação 193 implica por sua vez que a solução completa é:

$$
\begin{gathered}
v_{1}=\frac{1}{\sqrt{2 E} \sqrt{\sqrt{2}\left|p_{-}\right|}}\left(\begin{array}{c}
-p_{1}+i p_{2} \\
\sqrt{2} E \\
0 \\
-m
\end{array}\right), \\
v_{-1}=\frac{1}{\sqrt{2 E} \sqrt{\sqrt{2}\left|p_{-}\right|}}\left(\begin{array}{c}
-m \\
0 \\
\sqrt{2} E \\
p_{1}+i p_{2}
\end{array}\right) .
\end{gathered}
$$

O cálculo direto mostra também que a seguinte regra de soma rege para os espinores de polarização $v_{s}$ :

$$
\sum_{s= \pm 1} v_{s} \bar{v}_{s}=\frac{E \gamma^{+}+\left|p_{-}\right| \gamma^{-}+p_{\perp} \gamma^{\perp}-m}{\left|2 p_{-}\right|} .
$$




\section{Campo eletromagnético}

As equações do campo eletromagnético são as equações de Maxwell, que no espaço vazio se leem [60, 61] (em unidades com $c=1$, como temos determinado de início):

$$
\begin{gathered}
\nabla \cdot \boldsymbol{E}=0 ; \nabla \cdot \boldsymbol{B}=0 \\
\nabla \times \boldsymbol{E}+\frac{\partial \boldsymbol{B}}{\partial t}=\mathbf{0} ; \nabla \times \boldsymbol{B}-\frac{\partial \boldsymbol{E}}{\partial t}=\mathbf{0} .
\end{gathered}
$$

Em função do «tensor de Faraday» $F_{\mu \nu}$, definido da forma seguinte:

$$
\left[F_{\mu \nu}\right]:=\left(\begin{array}{cccc}
0 & E_{x} & E_{y} & E_{z} \\
-E_{x} & 0 & -B_{z} & B_{y} \\
-E_{y} & B_{z} & 0 & -B_{x} \\
-E_{z} & -B_{y} & B_{x} & 0
\end{array}\right)=-\left[F_{\nu \mu}\right],
$$

as equações de Maxwell no vácuo se escrevem:

$$
\partial_{\mu} F^{\mu \nu}=0, \partial_{\rho} F_{\mu \nu}+\partial_{\mu} F_{\nu \rho}+\partial_{\nu} F_{\rho \mu}=0,
$$

essa última conhecida como «identidade de Bianchi». Essas equações permitem a introdução do «potencial eletromagnético», $A_{\mu}(x)$, segundo:

$$
F_{\mu \nu}=: \partial_{\mu} A_{\nu}-\partial_{\nu} A_{\mu} .
$$

A este campo chamaremos doravante «campo eletromagnético», ou às vezes -para explicitar que ele será considerado livre - «campo da radiação». Ao passo que a identidade de Bianchi se mantém idênticamente satisfeita, a equação do movimento do campo eletromagnético [primeira das equações (230]] se reescreve:

$$
\square A^{\nu}(x)-\partial^{\nu}\left(\partial_{\mu} A^{\mu}(x)\right)=0,
$$

que pode ser obtida da densidade lagrangiana:

$$
\mathscr{L}_{A}=-\frac{1}{4} F_{\mu \nu} F^{\mu \nu} \text {. }
$$

\subsection{Componentes dinâmicas e não-dinâmicas. Condição de gauge do plano nulo}

Poderia parecer, olhando para a equação (232), que o campo de radiação não está obrigado a satisfazer a equação de Klein-Gordon-Fock. No entanto, o termo adicional pode ser removido pela imposição de uma condição subsidiária (condição de gauge ou de calibre) cuja possibilidade se manifesta pela não unicidade da relação entre o potencial e os campos $\boldsymbol{E}$ e $\boldsymbol{B}$ : Estes não são modificados se o potencial é redefinido pela adição de um termo de divergência, $A_{\mu} \rightarrow A_{\mu}+\partial_{\mu} f \sqrt{15}$ Para tal remoção bastará exigir que seja $\partial^{\nu}\left(\partial_{\mu} A^{\mu}\right)=0$, que é o mesmo que $\partial_{\mu} A^{\mu}=$ constante; impondo então que o campo se anule no infinito, a constante deve por força

\footnotetext{
15 Mais detalhes podem ser encontrados, por exemplo, na seção 18 das Refs. 60, 61.
}

ser nula e, assim, a equação de Klein-Gordon-Fock é obedecida sob a «condição de gauge de Lorenz»

$$
\partial_{\mu} A^{\mu}(x)=\partial_{+} A^{+}+\partial_{\perp} A^{\perp}+\partial_{-} A^{-}=0 .
$$

Essa condição de gauge elimina um grau de liberdade da teoria, mas não elimina por completo a liberdade de gauge, pois ainda a equação de Klein-Gordon-Fock e a condição de gauge de Lorenz serão satisfeitas simultâneamente caso se opere uma transformação de gauge com uma função harmônica ${ }^{17} \square f=0$. Para eliminar por completo a liberdade de gauge deve ser imposta uma condição adicional; a simplificação máxima na dinâmica da frente de luz consiste em tornar a condição de gauge de Lorenz numa relação puramente cinemática, eliminando toda dependência temporal nela; para tal será suficiente exigir que seja $\partial_{+} A^{+}=0$, mas então a equação $\square A^{+}=0$ será igual a $\partial_{\perp}^{2} A^{+}=0$, cuja solução, sob as condições assintóticas de desvanecimento no infinito, é a nula. A essa se chama a «condição de gauge do plano nulo» 18

$$
A^{+}(x)=0 \text {. }
$$

Isto elimina toda a liberdade de gauge, mostrando que o campo eletromagnético possue dois graus de liberdade. Efetivamente, toda a informação está contida nas equações dinâmicas para as componentes transversais:

$$
\square A^{\alpha}(x)=0,
$$

e nas equações de vínculo que provêm das condições de gauge [equações 234 e (235)]:

$$
A^{+}=0, A^{-}=-\frac{1}{\partial_{-}} \partial_{\alpha} A^{\alpha} .
$$

As componentes transversais $A^{\alpha}$ são chamadas «componentes dinâmicas» do campo de radiação, enquanto que $A^{+}$e $A^{-}$são as «não-dinâmicas», por estarem totalmente determinadas pelos vínculos.

É útil mencionar adicionalmente que, como afirmado nas Refs. [14, 15, 24, a análise que fizemos antes do problema de Goursat [vide a seção 3] pode ser fácilmente extendida para um campo $\Phi(x)$ que obedece a equações do tipo:

$$
\partial_{+} \partial_{-} \Phi(x)=L \Phi(x)+\Psi(x)
$$

com $L$ um operador diferencial linear que não contém derivadas temporais $\partial_{+}$e $\Psi(x)$ um campo diferente do

\footnotetext{
16 Essa condição de gauge é sempre atingível: Seja $g(x)=$ $\partial_{\mu} A^{\mu}(x)$. O campo $A^{\prime \mu}=A^{\mu}+\partial^{\mu} f$ satisfará à condição de gauge de Lorenz se a função $f(x)$ é escolhida de forma a satisfazer a equação: $\square f(x)=-g(x)$.

$17 \mathrm{Na}$ nota de rodapé anterior, a função $f(x)$ está determinada módulo soluções à equação homogênea $\square f(x)=0$.

18 É mister mencionar também que ela é sempre atingível: Se o campo $A^{a}(x)$ não a satisfaz, operamos a transformação $A^{\prime a}(x)=$ $A^{a}(x)+\partial^{a} f(x)$, com: $f(x)=-\frac{1}{\partial_{-}} A^{+}(x)$. Então a equação 235 rege para o campo $A^{\prime a}(x)$.
} 
$\Phi(x)$. As equações (232 são desse tipo para $\nu=1,2$ somente sob a condição de gauge do plano nulo. A equação para $\nu=+$ se equivale então à condição de gauge de Lorenz com $A^{+}=0$-sob as condições assintóticas que estamos usando-, e esta torna uma identidade a equação para $\nu=-$. As duas condições de gauge, de qualquer forma, são compatíveis - no caso livre- e devem ser usadas simultâneamente.

No gauge do plano nulo, a densidade lagrangiana da equação 233 se simplifica a:

$$
\begin{aligned}
\mathscr{L}_{A}= & \partial_{+} A^{\perp} \partial_{-} A^{\perp}+\partial_{-} A^{\perp} \partial_{\perp} A^{-} \\
& -\frac{1}{2}\left(\partial_{1} A^{2}-\partial_{2} A^{1}\right)^{2}+\frac{1}{2}\left(\partial_{-} A^{-}\right)^{2} .
\end{aligned}
$$

Ou ainda, em função das componentes dinâmicas só:

$$
\begin{aligned}
\mathscr{L}_{A}= & \partial_{+} A^{\perp} \partial_{-} A^{\perp}+\left(A^{\alpha} \partial_{\alpha}\right)\left(\partial_{\beta} A^{\beta}\right) \\
& +\frac{1}{2}\left(\partial_{\perp} A^{\perp}\right)^{2}-\frac{1}{2}\left(\partial_{1} A^{2}-\partial_{2} A^{1}\right)^{2} .
\end{aligned}
$$

O tensor de energia-momento é:

$$
\begin{aligned}
\Theta^{\mu}{ }_{\nu} & =\frac{\partial \mathscr{L}_{A}}{\partial\left(\partial_{\mu} A^{\sigma}\right)} \partial_{\nu} A^{\sigma}-\delta_{\nu}^{\mu} \mathscr{L}_{A} \\
& =F_{\sigma}{ }^{\mu} \partial_{\nu} A^{\sigma}+\frac{1}{4} \delta^{\mu}{ }_{\nu} F_{\sigma \tau} F^{\sigma \tau} .
\end{aligned}
$$

Particulamente, o hamiltoniano invariante no gauge do plano nulo é:

$$
\begin{aligned}
H_{N P ; A}= & \int d^{3} \underline{\boldsymbol{x}}\left[\frac{1}{2}\left(\partial_{1} A^{2}-\partial_{2} A^{1}\right)^{2}\right. \\
& \left.-\partial_{-} A^{\perp} \partial_{\perp} A^{-}-\frac{1}{2}\left(\partial_{-} A^{-}\right)^{2}\right] .
\end{aligned}
$$

Ou, escrito somente em função das componentes dinâmicas:

$$
H_{N P ; A}=\int d^{3} \underline{\boldsymbol{x}}\left[\frac{1}{2}\left(\partial_{1} A^{2}-\partial_{2} A^{1}\right)^{2}+\frac{1}{2}\left(\partial_{\perp} A^{\perp}\right)^{2}\right] .
$$

\subsection{Problema de Goursat}

O problema de Goursat para o campo eletromagnético possui uma consequência notável: Ele garante a preservação no tempo das condições de gauge. Com efeito, dada a equação 236, as componentes transversais têm a solução:

$$
A^{\alpha}(x)=\int_{y^{+}=y_{0}^{+}} d^{3} \underline{\boldsymbol{y}} D_{0}(x-y) \overleftrightarrow{\partial}_{-}^{y} A^{\alpha}(y)
$$

com o subíndice «0» sob a distribuição de Jordan-Pauli indicando que nela a massa é nula: $m=0$. Escrevamos a solução completa da forma:

$$
A^{a}(x)=\int_{y^{+}=y_{0}^{+}} d^{3} \underline{\boldsymbol{y}}_{b}^{a}{ }_{b}(x-y) \overleftrightarrow{\partial}_{-}^{y} A^{b}(y)
$$

A equação 244 é recuperada sob a condição de ser:

$$
D^{\alpha}{ }_{\beta}=\delta_{\beta}^{\alpha} D_{0}, D_{-}^{\alpha}=0,
$$

enquanto que as condições de vínculo da equação (237) são satisfeitas, uma vez impostas no plano nulo $y^{+}=y_{0}^{+}$, se:

$$
\begin{gathered}
D_{\alpha}^{+}=0, D_{-}^{+}=0, \\
D_{-}^{-}=0, D^{-}{ }_{\alpha}=-\frac{1}{\partial_{-}} \partial_{\alpha} D_{0} .
\end{gathered}
$$

Nota-se que não é preciso exigirmos valores particulares de $D^{a}{ }_{+}$, uma vez que imporemos que seja $A^{+}=0$ no plano nulo inicial. Todas essas condições são satisfeitas com a escolha:

$$
\begin{gathered}
D_{b}^{a}=\left(\delta_{b}^{a}-\frac{\eta^{a} \partial_{b}+\eta_{b} \partial^{a}}{\partial_{-}}\right) D_{0}(x), \\
\left(\eta^{a}\right)=\left(0 ; 0^{\perp} ; 1\right) .
\end{gathered}
$$

Assim, se no plano $y^{+}=y_{0}^{+}$são impostas as condições de gauge de Lorenz e do plano nulo, então elas se manterão em todo tempo posterior na evolução dinâmica do campo.

\subsection{Vetores de polarização}

Já dissemos repetidamente que sob a condição de gauge de Lorenz cada componente do campo da radiação satisfaz a equação de Klein-Gordon-Fock:

$$
\square A^{a}(x)=0 .
$$

Consequentemente, cada uma dessas componentes poderá ser expandida como uma integral de Fourier como na equação (179):

$$
\begin{aligned}
A^{a}(x)= & (2 \pi)^{-3 / 2} \int \frac{d^{3} \underline{\underline{p}}}{\sqrt{\left|2 p_{-}\right|}} \Theta\left(p_{-}\right) \\
& \times \sum_{\lambda}\left(\varepsilon_{\lambda}(\underline{\boldsymbol{p}})^{a *} A(\lambda ; \underline{\boldsymbol{p}}) e^{-i p x}\right. \\
& \left.+\varepsilon_{\lambda}(\underline{\boldsymbol{p}})^{a} A(\lambda ; \underline{\boldsymbol{p}})^{*} e^{i p x}\right)\left.\right|_{p_{+}=E},
\end{aligned}
$$

$\operatorname{com} \varepsilon_{\lambda}(\underline{\boldsymbol{p}})^{a}$ os vetores de polarização do campo $(\lambda=$ $+, \perp,-)$. Se eles são escolhidos reais, então a equação anterior se reduz à:

$$
\begin{aligned}
A^{a}(x)= & (2 \pi)^{-3 / 2} \int \frac{d^{3} \underline{\underline{p}}}{\sqrt{\left|2 p_{-}\right|}} \Theta\left(p_{-}\right) \sum_{\lambda} \varepsilon_{\lambda}(\underline{\boldsymbol{p}})^{a} \\
& \times\left.\left(A(\lambda ; \underline{\boldsymbol{p}}) e^{-i p x}+A(\lambda ; \underline{\boldsymbol{p}})^{*} e^{i p x}\right)\right|_{p_{+}=E} .
\end{aligned}
$$

O caráter vetorial do campo eletromagnético está, então, contido nos vetores de polarização e, portanto, 
(da mesma forma que acontecia com os espinores de polarização do campo de Dirac) devem carregar também a informação relativa aos vínculos impostos. Ora, como este campo possui somente dois graus de liberdade, também serão só dois os vetores de polarização dinâmicos; diremos que estes são aqueles com $\lambda=1,2$. As duas condições de gauge explicitadas na equação 237 são obtidas pelo uso dos vetores de polarização:

$$
\varepsilon_{1}(\underline{\boldsymbol{p}})^{a}=\left(0 ; 1 ; 0 ;-\frac{p_{1}}{p_{-}}\right), \varepsilon_{2}(\underline{\boldsymbol{p}})^{a}=\left(0 ; 0 ; 1 ;-\frac{p_{2}}{p_{-}}\right)
$$

cuja norma é:

$$
\eta_{a b} \varepsilon_{\alpha}(\underline{\boldsymbol{p}})^{a} \varepsilon_{\beta}(\underline{\boldsymbol{p}})^{b}=-\delta_{\beta}^{\alpha},
$$

o que indica que estão normalizados e são do tipoespaço. O cálculo direto então mostra que tais vetores de polarização físicos seguem a regra de soma:

$$
\sum_{\lambda=1,2} \varepsilon_{\lambda}(\underline{\boldsymbol{p}})^{a} \varepsilon_{\lambda}(\underline{\boldsymbol{p}})^{b}=-\eta^{a b}+\frac{p^{a} \eta^{b}+\eta^{a} p^{b}}{p_{-}}-\frac{p^{2}}{p_{-}^{2}} \eta^{a} \eta^{b} .
$$

Isto estabelece uma relação importante com o problema de Goursat: Devido à igualdade $p^{2} \delta\left(p^{2}\right)=0$ [62], as equações 83 e 248 implicam que, no espaço dos momentos:

$$
\widehat{D}^{a b}(p)=-\sum_{\lambda=1,2} \varepsilon_{\lambda}(\underline{\boldsymbol{p}})^{a} \varepsilon_{\lambda}(\underline{\boldsymbol{p}})^{b} \widehat{D}_{0}(p) .
$$

Devemos finalmente nos ocupar da definição dos outros dois vetores de polarização, embora eles não se manifestem fisicamente. Como o conjunto dos quatro vetores hão de formar uma base para o espaço-tempo de Minkowski, requeriremos sua ortonormalidade -que é uma extensão da equação 254 -:

$$
\eta_{a b} \varepsilon_{\lambda}(\underline{\boldsymbol{p}})^{a} \varepsilon_{\lambda^{\prime}}(\underline{\boldsymbol{p}})^{b}=\eta_{\lambda \lambda^{\prime}}
$$

equivalente à relação de completeza:

$$
\sum_{\lambda \lambda^{\prime}} \eta_{\lambda \lambda^{\prime}} \varepsilon_{\lambda}(\underline{\boldsymbol{p}})^{a} \varepsilon_{\lambda^{\prime}}(\underline{\boldsymbol{p}})^{b}=\eta^{a b} .
$$

Digamos que sejam

$$
\varepsilon_{+}(\underline{\boldsymbol{p}})^{a}=(a ; b ; c ; d), \varepsilon_{-}(\underline{\boldsymbol{p}})^{a}=(e ; f ; g ; h) .
$$

As condições de serem $\varepsilon_{+}(\underline{\boldsymbol{p}})_{a} \varepsilon_{+}(\underline{\boldsymbol{p}})^{a}=0, \varepsilon_{1}(\underline{\boldsymbol{p}})_{a}$ $\varepsilon_{+}(\underline{\boldsymbol{p}})^{a}=0$ e $\varepsilon_{2}(\underline{\boldsymbol{p}})_{a} \varepsilon_{+}(\underline{\boldsymbol{p}})^{a}=0$ levam a que, quer $a \neq \overline{0} \mathrm{e}$

$$
\varepsilon_{+}(\underline{\boldsymbol{p}})^{a}=a\left(1 ;-\frac{p_{1}}{p_{-}} ;-\frac{p_{2}}{p_{-}} ; \frac{p_{\perp}^{2}}{2 p_{-}^{2}}\right),
$$

quer $a=0$ e

$$
\varepsilon_{+}(\underline{\boldsymbol{p}})^{a}=(0 ; 0 ; 0 ; d) .
$$

Analogamente, as condições de serem $\varepsilon_{-}(\underline{\boldsymbol{p}})_{a} \varepsilon_{-}(\underline{\boldsymbol{p}})^{a}=$ $0, \varepsilon_{1}(\underline{\boldsymbol{p}})_{a} \varepsilon_{-}(\underline{\boldsymbol{p}})^{a}=0$ e $\varepsilon_{2}(\underline{\boldsymbol{p}})_{a} \varepsilon_{-}(\underline{\boldsymbol{p}})^{a}=0$ implicam que, quer $e \neq 0$ e

$$
\varepsilon_{-}(\underline{\boldsymbol{p}})^{a}=e\left(1 ;-\frac{p_{1}}{p_{-}} ;-\frac{p_{2}}{p_{-}} ; \frac{p_{\perp}^{2}}{2 p_{-}^{2}}\right),
$$

quer $e=0 \mathrm{e}$

$$
\varepsilon_{-}(\underline{\boldsymbol{p}})^{a}=(0 ; 0 ; 0 ; h)
$$

Evidentemente, somente poderá ser $a \neq 0$ e $e=0$, ou $a=0$ e $e \neq 0$, pois de outra forma os vetores serão proporcionais um ao outro. Qual dessas duas possibilidades deve ser usada, é impossível dizê-lo; é matéria de escolha. Escolheremos a primeira. Então a condição de ser $\varepsilon_{+}(\underline{\boldsymbol{p}})_{a} \varepsilon_{-}(\underline{\boldsymbol{p}})^{a}=1$ implica que: $a h=1$, cuja mais simples solução é: $a=1=h$. Os vetores de polarização não-dinâmicos serão portanto:

$$
\begin{gathered}
\varepsilon_{+}(\underline{\boldsymbol{p}})^{a}=\left(1 ;-\frac{p_{1}}{p_{-}} ;-\frac{p_{2}}{p_{-}} ; \frac{p_{\perp}^{2}}{2 p_{-}^{2}}\right), \\
\varepsilon_{-}(\underline{\boldsymbol{p}})^{a}=(0 ; 0 ; 0 ; 1) .
\end{gathered}
$$

\section{Campo vetorial massivo}

Por fim, consideremos o campo vetorial massivo rea ${ }^{19}$ $B^{a}(x)$, e estabeleçamos para ele a densidade lagrangiana de Proca:

$$
\mathscr{L}_{B}=-\frac{1}{4} F_{a b} F^{a b}+m^{2} B_{a} B^{a},
$$

com:

$$
F_{a b}:=\partial_{a} B_{b}-\partial_{b} B_{a}
$$

As equações do movimento do campo são, portanto:

$$
\partial_{b} F^{b a}+m^{2} B^{a}=0,
$$

e o tensor de energia-momento que lhe é associado:

$$
\Theta^{\mu}{ }_{\nu}=F_{\sigma}{ }^{\mu} \partial_{\nu} B^{\sigma}+\frac{1}{4} \delta_{\nu}^{\mu} F_{\sigma \tau} F^{\sigma \tau}-\frac{1}{2} \delta_{\nu}^{\mu} m^{2} B_{\sigma} B^{\sigma} .
$$

\subsection{Problema de Goursat}

Nota-se, contudo, que o tensor $F^{a b}$ é antissimétrico, do que decorre que a aplicação da derivada $\partial_{a}$ à equação (268) leva imediatamente ao cumprimento da condição:

$$
\partial_{a} B^{a}(x)=0
$$

\footnotetext{
19 Não se deve pensar que essa seja uma restrição, pois bem se conhece que o campo complexo pode ser considerado como uma combinação linear complexa de dois campos reais; a análise só requeriria incrementar o número de campos reais seguindo o mesmo procedimento que aqui mostraremos para cada um deles.
} 
cuja substituição na equação 268 implica que as equações do movimento são as equações de Klein-GordonFock de cada uma das componentes:

$$
\left(\square+m^{2}\right) B^{a}(x)=0 .
$$

O conjunto das equações 270 e (271) é assim equivalente à equação 268. Em consequência, como tais componentes estão relacionadas pelo vínculo da equação (270), somente três componentes do campo vetorial massivo serão independentes, enquanto que a outra será determinada por eles. Mas a diferença do campo eletromagnético, como a densidade lagrangiana não possui a invariância de calibre, nenhuma outra condição subsidiária pode ser imposta; em particular, a componente $B^{+}(x)$ não pode ser anulada, e o vínculo não é cinemático, pois inclui uma derivada temporal. Assim, o vínculo bem pode ser entendido como uma equação dinâmica para a componente $B^{+}(x)$ :

$$
\partial_{+} B^{+}(x)=-\partial_{\alpha} B^{\alpha}(x)-\partial_{-} B^{-}(x) .
$$

Essa equação não substitui a equação de Klein-GordonFock para a componente $B^{+}$, pois ela não é identicamente satisfeita, uma vez que $B^{\alpha}, B^{-}$verificam a mencionada equação. Em seu lugar, o vínculo torna essa em uma equação não-dinâmica:

$$
\left(\partial_{\perp}^{2}-m^{2}\right) B^{+}(x)=-2 \partial_{-}\left(\partial_{\alpha} B^{\alpha}+\partial_{-} B^{-}\right),
$$

cuja solução é única uma vez impostas as condições assintóticas de desvanecimento de $B^{+}(x)$ no infinito das variáveis $x^{\perp}$. Em particular, é claro que as condições assintóticas tanto nessas direções como na direção da variável $x^{-}$serão implicadas por aquelas impostas às componentes $B^{\alpha}$ e $B^{-}$. Portanto, não é necessário fornecer dados iniciais para a componente $B^{+}$, que são consequência da equação 273.

Com a análise anterior provamos que o número de dados iniciais requeridos é igual a seis: Os valores das componentes $B^{\alpha}$ e $B^{-}$nas superfícies características -ou na superfície inicial e condições assintóticas na variável $x^{-}-$. A solução ao problema de Goursat pode então ser escrita da seguinte forma:

$$
\begin{aligned}
B^{+}(x)= & B^{+}\left(x_{0}^{+} ; \underline{\boldsymbol{x}}\right) \\
& -\int_{x_{0}^{+}}^{x^{+}} d y^{+}\left(\partial_{\alpha} B^{\alpha}+\partial_{-} B^{-}\right)\left(y^{+} ; \underline{\boldsymbol{x}}\right), \\
B^{\alpha,-}(x)= & \int_{y^{+}=x_{0}^{+}} d^{3} \underline{\boldsymbol{y}} D(x-y) \overleftrightarrow{\partial^{y}} B^{\alpha,-}(y),
\end{aligned}
$$

com $B^{+}\left(x_{0}^{+}, \underline{\boldsymbol{x}}\right)$ a solução à equação 273 em $x^{+}=x_{0}^{+}$. Contudo, essa forma da solução tem a desagradável desvantagem de não ser covariante. Por esse motivo, é preferível escrever a solução da seguinte forma:

$$
B^{a}(x)=\int_{y^{+}=x_{0}^{+}} d^{3} \underline{\boldsymbol{y}}_{D^{a}}^{a}(x-y) \overleftrightarrow{\partial}_{-}^{y} B^{b}(y)
$$

com $D^{a}{ }_{b}(x)$ escolhido de sorte que as equações 270 e (271) sejam satisfeitas. Evidentemente, para isso é suficiente que sejam cumpridas: $\left(\square+m^{2}\right) D_{b}^{a}(x)=0$ e $\partial_{a} D_{b}^{a}(x)=0$, o que é verificado por:

$$
D_{b}^{a}(x)=\left(\delta_{b}^{a}+\frac{\partial^{a} \partial_{b}}{m^{2}}\right) D(x)
$$

A forma assim escrita é compatível com o número de dados iniciais requeridos: Embora pareça que precisamos conhecer $B^{+}$na superfície inicial para colocá-la no lado direito da equação (276), ela ainda pode ser determinada com o mero conhecimento de $B^{\alpha}$ e $B^{-}$em dita superfície, pois as equações 276 e (277) ainda implicam a equação 273 .

\subsection{Vetores de polarização}

Como cada componente do campo vetorial massivo satisfaz a equação de Klein-Gordon-Fock, poderemos escrever, já escolhendo os vetores de polarização $\varepsilon_{\lambda}(\underline{\boldsymbol{p}})^{a}$ reais:

$$
\begin{aligned}
B^{a}(x)= & (2 \pi)^{-3 / 2} \int \frac{d^{3} \underline{\underline{p}}}{\sqrt{\left|2 p_{-}\right|}} \Theta\left(p_{-}\right) \sum_{\lambda} \varepsilon_{\lambda}(\underline{\boldsymbol{p}})^{a} \\
& \times\left.\left(B(\lambda ; \underline{\boldsymbol{p}}) e^{-i p x}+B(\lambda ; \underline{\boldsymbol{p}})^{*} e^{i p x}\right)\right|_{p_{+}=E} .
\end{aligned}
$$

Como outrora fizemos para o campo eletromagnético, é mister também agora definir os vetores de polarização com a finalidade de satisfazer aos vínculos impostos. Para satisfazer ao vínculo $\partial_{a} B^{a}(x)=0$ é necessário escolher os três vetores de polarização físicos, que denotaremos $\varepsilon_{j}(\underline{p})^{a}(j=1,2,3)$, tais que:

$$
p_{a} \varepsilon_{j}(\underline{\boldsymbol{p}})^{a}=0 \text {. }
$$

Daqui, os vetores de polarização físicos deverão ser da forma:

$$
\varepsilon_{j}(\underline{\boldsymbol{p}})^{a}=\left(\delta_{b}^{a}-\frac{p^{a} p_{b}}{m^{2}}\right) \theta_{j}(\underline{\boldsymbol{p}})^{b},
$$

com $\theta_{j}(\boldsymbol{p})^{b}$ vetores linearmente independentes escolhidos de forma que a norma dos vetores de polarização seja igual à unidade. Claramente, desde que o momento $p^{a}$ é do tipo-tempo - por se tratar de um campo massivo-, os vetores de polarização serão do tipo-espaço, e então sua norma, exigiremos, é igual a -1 . Escolhemos portanto os três vetores $\theta_{j}^{a}$ na direção dos eixos espaciais ${ }^{20}$

$$
\begin{gathered}
\theta_{1}^{a} \sim(0 ; 1 ; 0 ; 0), \quad \theta_{2}^{a} \sim(0 ; 0 ; 1 ; 0), \\
\theta_{3}^{a} \sim(1 ; 0 ; 0 ;-1) .
\end{gathered}
$$

\footnotetext{
${ }^{20}$ Essa é apenas uma escolha na multiplicidade de possibilidades.
} 
Os vetores de polarização normalizados que obtemos são:

$$
\begin{aligned}
& \varepsilon_{1}(\underline{\boldsymbol{p}})^{a}=\left(1+\frac{p_{1}^{2}}{m^{2}}\right)^{-1} \\
& \times\left(-\frac{p_{-} p_{1}}{m^{2}} ; 1+\frac{p_{1}^{2}}{m^{2}} ; \frac{p_{2} p_{1}}{m^{2}} ;-\frac{p_{+} p_{1}}{m^{2}}\right), \\
& \varepsilon_{2}(\underline{\boldsymbol{p}})^{a}=\left(1+\frac{p_{2}^{2}}{m^{2}}\right)^{-1} \\
& \times\left(-\frac{p_{-} p_{2}}{m^{2}} ; \frac{p_{1} p_{2}}{m^{2}} ; 1+\frac{p_{2}^{2}}{m^{2}} ;-\frac{p_{+} p_{2}}{m^{2}}\right), \\
& \varepsilon_{3}(\underline{\boldsymbol{p}})^{a}=\left(1+\frac{\left(p_{+}-p_{-}\right)^{2}}{2 m^{2}}\right)^{-1} \\
& \times\left(\frac{1}{\sqrt{2}}-\frac{p_{-}\left(p_{+}-p_{-}\right)}{\sqrt{2} m^{2}} ; \frac{p_{1}\left(p_{+}-p_{-}\right)}{\sqrt{2} m^{2}} ;\right. \\
& \left.\frac{p_{2}\left(p_{+}-p_{-}\right)}{\sqrt{2} m^{2}} ;-\frac{1}{\sqrt{2}}-\frac{p_{+}\left(p_{+}-p_{-}\right)}{\sqrt{2} m^{2}}\right) \text {. }
\end{aligned}
$$

Estes três vetores geram o espaço tri-dimensional ortogonal ao momento $p^{a}$. O vetor de polarização não físico, evidentemente, deverá ser definido ao longo de tal vetor:

$$
\varepsilon_{0}(\underline{\boldsymbol{p}})^{a}=\frac{p^{a}}{m}
$$

A regra de soma para os vetores de polarização físicos é agora:

$$
\sum_{j=1}^{3} \varepsilon_{j}(\underline{\boldsymbol{p}})^{a} \varepsilon_{j}(\underline{\boldsymbol{p}})^{b}=-\eta^{a b}+\frac{p^{a} p^{b}}{m^{2}} .
$$

Como alhures encontramos para o campo eletromagnético, essa regra de soma está intimamente relacionada com o problema de Goursat: Comparando as equações 277 e 286 vemos que no espaço dos momentos:

$$
\widehat{D}^{a b}(p)=-\sum_{j=1}^{3} \varepsilon_{j}(\underline{\boldsymbol{p}})^{a} \varepsilon_{j}(\underline{\boldsymbol{p}})^{b} \widehat{D}(p) .
$$

\section{Discussão e conclusões}

Apresentamos, detalhadamente, a teoria de campos clássicos livres na dinâmica do plano nulo, surgida como a possibilidade mais conveniente, desde a perspectiva hamiltoniana, dentre as diversas formas dinâmicas relativísticas. Porém, vimos que os planos nulos são precisamente as superfícies características da equação de Klein-Gordon-Fock, constituíndo justamente o único caso em que sua solução é diferente daquela da dinâmica instantânea, pois a hipótese do teorema de CauchyKovalevskaya não se lhe aplica. Contudo, mostramos que esta possibilidade é de fato realizável para os diferentes campos: escalar, fermiônico, eletromagnético e vetorial massivo. Em todos os casos determinamos a solução única dependente apenas dos dados iniciais, que agora devem ser: o valor do campo na superfície inicial $x^{+}=$ constante e a condição assintótica de desvanecimento do campo no infinito d a v ariável $x-$. Encontramos também para estes campos o gerador de translações no tempo $x^{+}$(hamiltoniano no plano nulo) e fizemos um estudo a respeito de suas componentes dinâmicas e não dinâmicas, relacionadas sempre por equações de vínculo em virtude das quais foram determinados os vetores (ou espinores, no caso fermiônico) de polarização dos campos.

Com os procedimentos e resultados expostos temos estabelecido as bases da teoria de campos no plano nulo. A utilidade dela será encontrada, por exemplo, na teoria conforme. Tal estudo, assim como o das interações entre os campos no plano nulo, que apresentará peculiaridades devido à presença de termos «instantâneos», será abordado em um próximo artigo. Igualmente, temos como perspectiva abordar a quantização dos campos no plano nulo que aqui estudamos classicamente, visto que a teoria quântica de campos no plano nulo é uma ferramenta poderosa no estudo da física hadrônica.

\section{Agradecimentos}

O.A.A. agradece à CAPES pelo apoio financeiro total; K.P.G. e B.M.P. agradecem ao CNPq pelo apoio financeiro total e parcial, respectiva-mente. G.E.R.Z. agradece ao Sistema de Investigacio-nes Universidad de Nariño.

\section{A. Álgebra de Poisson das quantidades fundamentais}

Demonstraremos nesse apêndice que a álgebra das quantidades fundamentais é a álgebra do grupo de Poincaré. Consideremos primeiramente a ação de duas transformações consecutivas, caracterizadas pelos geradores $F_{1}$ e $F_{2}$. A variável dinâmica $\xi$ muda então para -mantendo termos até de ordem $F_{1} F_{2}$, mas desconsiderando as da ordem $F_{1}^{2}$ e $F_{2}^{2-}$ :

$$
\xi^{\prime \prime}=\xi+\left\{\xi ; F_{1}\right\}+\left\{\xi ; F_{2}\right\}+\left\{\left\{\xi ; F_{2}\right\} ; F_{1}\right\} .
$$

Se as transformações forem aplicadas na ordem inversa, isto é, primeiro $F_{2}$ e depois $F_{1}$, a variável dinâmica teria se tornado:

$$
\xi^{* *}=\xi+\left\{\xi ; F_{1}\right\}+\left\{\xi ; F_{2}\right\}+\left\{\left\{\xi ; F_{1}\right\} ; F_{2}\right\} .
$$

Dessas equações, a identidade de Jacobi expressa na equação (4) leva a que a diferença entre a aplicação das transformações em uma ordem ou em outra é igual a:

$$
\xi^{* *}-\xi^{\prime \prime}=\left\{\xi ;\left\{F_{1} ; F_{2}\right\}\right\} .
$$

Portanto, o comutador das pequenas variações da variável dinâmica se relaciona com o colchete de Poisson dos geradores das transformações correspondentes. Assim é que poderemos estabelecer a álgebra de colchetes de Poisson entre os geradores $P$ e $M$ das transformações 
de Poincaré, uma vez que conhecemos a ação deles nas coordenadas. Comecemos por ver que, segundo as equações (5), (6) e (7):

$$
a^{\mu}+b^{\mu \nu} x_{\nu}=-a^{\rho}\left\{x^{\mu} ; P_{\rho}\right\}+\frac{1}{2} b^{\rho \sigma}\left\{x^{\mu} ; M_{\rho \sigma}\right\},
$$

cuja solução é:

$$
\left\{x^{\mu} ; P_{\rho}\right\}=-\delta_{\rho}^{\mu}, \quad\left\{x^{\mu} ; M_{\rho \sigma}\right\}=\delta_{\rho}^{\mu} x_{\sigma}-\delta_{\sigma}^{\mu} x_{\rho} .
$$

Definamos agora os geradores:

$$
\begin{aligned}
& F_{1}=-P_{\mu} a^{\mu}+\frac{1}{2} M_{\mu \nu} b^{\mu \nu} \\
& F_{2}=-P_{\rho} a^{\prime \rho}+\frac{1}{2} M_{\rho \sigma} b^{\prime \rho \sigma}
\end{aligned}
$$

cujo comutador é:

$$
\begin{gathered}
\left\{F_{1} ; F_{2}\right\}=a^{\mu} a^{\rho}\left\{P_{\mu} ; P_{\rho}\right\}-\frac{1}{2} a^{\mu} b^{\prime \rho \sigma}\left\{P_{\mu} ; M_{\rho \sigma}\right\} \\
-\frac{1}{2} a^{\prime \rho} b^{\mu \nu}\left\{M_{\mu \nu} ; P_{\rho}\right\}+\frac{1}{4} b^{\mu \nu} b^{\prime \rho \sigma}\left\{M_{\mu \nu} ; M_{\rho \sigma}\right\} .
\end{gathered}
$$

Por outro lado, as transformações de coordenadas correspondentes, aplicadas sucessivamente, levam, via a equação (6), a:

$$
\begin{aligned}
x^{\prime \prime \tau}= & x^{\tau}+\left(a^{\prime \tau}+a^{\tau}+b^{\prime \tau \eta} a_{\eta}\right) \\
& +\left(b^{\tau \lambda}+b^{\prime \tau \lambda}+b^{\prime \tau}{ }_{\eta} b^{\eta \lambda}\right) x_{\lambda}, \\
x^{* * \tau}= & x^{\tau}+\left(a^{\tau}+a^{\prime \tau}+b^{\tau \eta} a_{\eta}^{\prime}\right) \\
& +\left(b^{\prime \tau \lambda}+b^{\tau \lambda}+b^{\tau}{ }_{\eta} b^{\prime \eta \lambda}\right) x_{\lambda},
\end{aligned}
$$

e obtemos:

$$
x^{* * \tau}-x^{\prime \prime \tau}=b^{\tau \eta} a_{\eta}^{\prime}-b^{\prime \tau \eta} a_{\eta}+\left(b_{\eta}^{\tau} b^{\prime \eta \lambda}-b^{\prime \tau}{ }_{\eta} b^{\eta \lambda}\right) x_{\lambda} .
$$

O lado direito dessa equação, tal como o ditam as equações 290 e 293, deverá igualarse a:

$$
\begin{aligned}
a^{\mu} a^{\rho} & \left\{x^{\tau} ;\left\{P_{\mu} ; P_{\rho}\right\}\right\}-\frac{1}{2} a^{\mu} b^{\prime \rho \sigma}\left\{x^{\tau} ;\left\{P_{\mu} ; M_{\rho \sigma}\right\}\right\} \\
& -\frac{1}{2} a^{\rho} b^{\mu \nu}\left\{x^{\tau} ;\left\{M_{\mu \nu} ; P_{\rho}\right\}\right\} \\
& +\frac{1}{4} b^{\mu \nu} b^{\prime \rho \sigma}\left\{x^{\tau} ;\left\{M_{\mu \nu} ; M_{\rho \sigma}\right\}\right\} .
\end{aligned}
$$

E dessa igualdade, por meio da equação 292, obtémse a álgebra de colchetes de Poisson dos geradores das transformações de Poincaré contida nas equações (8)- 10 .

\section{B. Matrizes de Dirac no plano nulo}

Na representação de Weyl as matrizes de Dirac são:

$$
\gamma^{0}=\left(\begin{array}{ll}
0_{2} & 1_{2} \\
1_{2} & 0_{2}
\end{array}\right), \gamma^{k}=\left(\begin{array}{cc}
0_{2} & -\sigma_{k} \\
\sigma_{k} & 0_{2}
\end{array}\right),
$$

com $\sigma_{k}$ as matrizes de Pauli:

$$
\sigma_{1}=\left(\begin{array}{ll}
0 & 1 \\
1 & 0
\end{array}\right), \sigma_{2}=\left(\begin{array}{cc}
0 & -i \\
i & 0
\end{array}\right), \sigma_{3}=\left(\begin{array}{cc}
1 & 0 \\
0 & -1
\end{array}\right) .
$$

Assim, temos que as matrizes $\gamma^{ \pm}$, definidas segundo:

$$
\gamma^{ \pm}:=\frac{1}{\sqrt{2}}\left(\gamma^{0} \pm \gamma^{3}\right)
$$

têm a seguinte forma explícita, na representação em uso:

$$
\gamma^{+}=\left(\begin{array}{cccc}
0 & 0 & 0 & 0 \\
0 & 0 & 0 & \sqrt{2} \\
\sqrt{2} & 0 & 0 & 0 \\
0 & 0 & 0 & 0
\end{array}\right), \gamma^{-}=\left(\begin{array}{cccc}
0 & 0 & \sqrt{2} & 0 \\
0 & 0 & 0 & 0 \\
0 & 0 & 0 & 0 \\
0 & \sqrt{2} & 0 & 0
\end{array}\right) \text {. }
$$

Os projetores $\Lambda_{ \pm}$definidos na equação 190 são portanto:

$$
\Lambda_{+}=\left(\begin{array}{cccc}
1 & 0 & 0 & 0 \\
0 & 0 & 0 & 0 \\
0 & 0 & 0 & 0 \\
0 & 0 & 0 & 1
\end{array}\right), \Lambda_{-}=\left(\begin{array}{llll}
0 & 0 & 0 & 0 \\
0 & 1 & 0 & 0 \\
0 & 0 & 1 & 0 \\
0 & 0 & 0 & 0
\end{array}\right)
$$

\section{Referências}

[1] A. Einstein, Ann. der Phys. 17, 891 (1905).

[2] H. Lorentz, A. Einstein e H. Minkowski, Textos Fundamentais da Física Moderna. I Volume: O Princípio da Relatividade (Fundação Calouste Gulbenkian, Lisboa, 2001), 5a ed.

[3] H. Poincaré, Science and Hypothesis (The Walter Scott publishing Co., Londres, 1905).

[4] A.A. Logunov, arXiv:physics/0408077 (2004).

[5] W. Rindler, Relativity. Special, General and Cosmological (Oxford University Press, Nova Iorque, 2006), 2a ed.

[6] P.A.M. Dirac, Rev. Mod. Phys. 21, 392 (1949).

[7] G.E. Ramos, Teoria de Campos no Plano Nulo: Um estudo. Dissertação de Mestrado, Universidade Estadual Paulista Júlio de Mesquita Filho, São Paulo (2005).

[8] G.E. Ramos, Formulação Canônica no Plano Nulo. Tese de Doutorado, Universidade Estadual Paulista Júlio de Mesquita Filho, São Paulo (2009).

[9] H. Bacry, P. Combe e P. Sorba, Rep. Math. Phys. 5, 145 (1974).

[10] H. Bacry, P. Combe e P. Sorba, Rep. Math. Phys. 5, 361 (1974).

[11] J. Patera, P. Winternitz e H. Zassenhaus, J. Math. Phys. 16, 1597 (1975).

[12] J. Patera, R. Sharp, P. Winternitz e H. Zassenhaus, J. Mark Phys. 17, 977 (1976).

[13] H. Leutwyler e J. Stern, Ann. Phys. 112, 94 (1978).

[14] F. Rohrlich, Acta Phys. Austriaca 32, 87 (1970).

[15] F. Rohrlich, Acta Phys. Austriaca Suppl. VIII, 277 (1971).

[16] S.J. Brodsky, H.C. Pauli e S.S. Pinsky, Phys. Rep. 301, 299 (1998).

[17] S. Fubini e G. Furlan, Physics Physique Fizika 1, 299 (1965).

[18] S. Weinberg, Phys. Rev. 150, 1313 (1966). 
[19] H. Bebié e H. Leutwyler, Phys. Rev. Lett. 19, 618 (1967)

[20] R.A. Neville, Quantum Electrodynamics in a Laser Pulse. Tese de Doutorado, Syracuse University, Syracuse (1968).

[21] R.A. Neville e F. Rohrlich, Phys. Rev. D 3, 1692 (1971).

[22] S.J. Chang e S. Ma, Phys. Rev. 180, 1506 (1969).

[23] J.B. Kogut e D.E. Soper, Phys. Rev. D 1, 2901 (1970).

[24] R.A. Neville e F. Rohrlich, Nuovo Cimento 1, 625 (1971).

[25] H. Leutwyler, J.R. Klauder e L. Streit, Nuovo Cimento LXVI A, 536 (1970).

[26] J.H. Ten Eyck, Problems in Null-Plane QuantumElectrodynamics. Tese de Doutorado, Syracuse University, Syracuse (1973).

[27] J.H. Ten Eyck e F. Rohrlich, Phys. Rev. D 9, 2237 (1974).

[28] S.J. Chang, R.G. Root e T.M. Yan, Phys. Rev. D 7, 1133 (1973).

[29] S.J. Chang e T.M. Yan, Phys. Rev. D 7, 1147 (1973).

[30] T.M. Yan, Phys. Rev. D 7, 1760 (1973).

[31] T.M. Yan, Phys. Rev. D 7, 1780 (1973).

[32] S.J. Brodsky, R. Roskies e R. Suaya, Phys. Rev. D 8 4574 (1973).

[33] S.D. Drell, D. Levy e T.M. Yan, Phys. Rev. 187, 2159 (1969).

[34] S.D. Drell, D. Levy e T.M. Yan, Phys. Rev. D 1, 1035 (1970).

[35] S.D. Drell, D. Levy e T.M. Yan, Phys. Rev. D 1, 1617 (1970).

[36] S.D. Drell e T.M. Yan, Phys. Rev. Lett. 24, 181 (1970).

[37] E.P. Biernat, W.H. Klink e W. Schweiger, Few-Body Syst. 49, 149 (2011).

[38] R. Casana, B.M. Pimentel e G.E.R Zambrano, Braz. J. Phys. 44, 398 (2014).

[39] T. Maskawa e K. Yamawaki, Progr. Theor. Phys. 56, 270 (1976).

[40] H.C. Pauli e S.J. Brodsky, Phys. Rev. D 32, 1993 (1985).

[41] H.C. Pauli e S.J. Brodsky, Phys. Rev. D 32, 2001 (1985).

[42] E. Tomboulis, Phys. Rev. D 8, 2736 (1973).

[43] A. Casher, Phys. Rev. D 14, 452 (1976).

[44] B.M. Pimentel, A.T. Suzuki e G.E.R. Zambrano, FewBody Syst. 52, 437 (2012).

[45] B.L.G. Bakker, A. Bassetto, S.J. Brodsky, W. Broniowski, S. Dalley, T. Frederico e T. Heinzl, Nuclear Phys. B (Proc. Suppl.) 251-252, 165 (2014).

[46] D. Bhamre, A. Misra e V.K. Singh, Few Body Syst. 59, 107 (2018).

[47] A.T. Suzuki e T.H.O. Sales, arXiv:hep-th/0408135 (2004).

[48] L. Landau e E. Lifshitz, Course of Theoretical Physics. Vol. 1. Mechanics (Butterworth-Heinemann, Oxford, 1976), 3 ed.

[49] L. Landau e E. Lifchitz, Curso de Física. Mecânica (Hemus, São Paulo, 2002).

[50] C. Lämmerzahl, J. Math. Phys. 34, 3918 (1993).

[51] J. Hadamard, Lectures on Cauchy's Problem in Linear Partial Differential Equations (Yale University Press, New Haven, 1923).

[52] E. Goursat, A Course in Mathematical Analysis. Vol. I (Ginn and Company, Boston, 1904).
[53] N.N. Bogoliubov e D. Shirkov, Introduction to the theory of Quantized Fields (John Wiley \& Sons - Interscience Publishers, Nova Iorque, 1979), 3 ed.

[54] A.N. Tíjonov e A.A. Samarsky, Ecuaciones de la física matemática (Editorial Mir Moscú, Moscou, 1972).

[55] S. Chandrasekhar, The Mathematical Theory of Black Holes (Oxford University Press, Oxford, 2006).

[56] E. Gourgoulhon, Special Relativity in General Frames. From Particles to Astrophysics (Springer-Verlag, Heidelberg, 2013).

[57] F.G. Friedlander e M. Joshi, Introduction to the theory of distributions (Cambridge University Press, Cambridge, 1998), 2 ed.

[58] A.G. Sveshnikov e A.N. Tikhonov, The Theory of Functions of a Complex Variable (Mir Publishers, Moscou, 1971).

[59] N.P. Konopleva e V.N. Popov, Gauge Fields (Harwood academic publishers, Amsterdã, 1981).

[60] L. Landau e E. Lifshitz, Course of Theoretical Physics. Vol. 2. The Classical Theory of Fields (ButterworthHeinemann, Oxford, 1980), 4 ed.

[61] L. Landau e E. Lifchitz, Curso de Física. Teoria do campo (Hemus, São Paulo, 2002).

[62] L. Schwartz, Mathematics for the Physical Sciences (Addison-Wesley Publishing Company, Paris, 1966), 2 ed.

[63] J. Hilgevoord, Dispersion Relations and Causal Description (North Holland, Amsterdã, 1960).

[64] R. Casana, B.M. Pimentel e G.E.R. Zambrano, Revista Colombiana de Física 41, 220 (2009). 